UPR-669

\title{
Front Propagation in the Pearling Instability of Tubular Vesicles
}

\author{
Raymond E. Goldstein \\ Joseph Henry Laboratory of Physics, Princeton University \\ Princeton, NJ 08544 USA \\ Philip Nelson and Thomas Powers* \\ Physics Department, University of Pennsylvania \\ Philadelphia, PA 19104 USA \\ Udo Seifert \\ Max-Planck-Institut für Kolloid- und Grenzflächenforschung \\ Kantstr. 55, 14513 Teltow-Seehof, Germany
}

\begin{abstract}
Recently Bar-Ziv and Moses discovered a dynamical shape transformation induced in cylindrical lipid bilayer vesicles by the action of laser tweezers. We develop a hydrodynamic theory of fluid bilayers in interaction with the surrounding water and argue that the effect of the laser is to induce a sudden tension in the membrane. We refine our previous analysis to account for the fact that the shape transformation is not uniform but propagates outward from the laser trap. Applying the marginal stability criterion to this situation gives us an improved prediction for the selected initial wavelength and a new prediction for the propagation velocity, both in rough agreement with the experimental values. For example, a tubule of initial radius $0.7 \mu \mathrm{m}$ has a predicted initial sinusoidal perturbation in its diameter with wavelength $5.5 \mu \mathrm{m}$, as observed. The perturbation propagates as a front with the qualitatively correct front velocity a bit less than $100 \mu \mathrm{m} / \mathrm{sec}$. In particular we show why this velocity is initially constant, as observed, and so much smaller than the natural scale set by the tension. We also predict that the front velocity should increase linearly with laser power. Finally we introduce an approximate hydrodynamic model applicable to the fully nonlinear regime. This model exhibits propagating fronts as well as fully-developed "pearled" vesicles similar to those seen in the experiments.
\end{abstract}

$9 / 95$

* Present address: Dept. of Physics, Princeton University, Princeton, NJ 08544 and NEC Research Institute, 4 Independence Way, Princeton, NJ 08540. 


\section{Introduction and Summary}

The study of artificial biomembranes has opened a window into the machinery of real cells by giving us physical systems which are simple enough to describe from first principles, yet complicated enough to display lifelike behavior. The study of the equilibrium configurations of closed bags of lipid bilayers ("vesicles") is by now well advanced [1][2]. Most interesting biophysical phenomena, however, are not in equilibrium, and the study of the dynamics of vesicle shapes is somewhat less developed. For example, budding and vesiculation [3] and instabilities crucial for understanding adhesion [4], are all inherently dynamical processes.

To gain a physical understanding of non-equilibrium membrane dynamics we must begin with experiments in which some physical intervention brings about a dynamical shape transformation.1 Several such techniques are by now well developed. Budding and other shape transformations can be induced by adjusting temperature in closed vesicles [5] [6]. Strings of beads can be induced by relieving tension using micropipettes [7], by sudden hydration of dry lipid [8], and by the incorporation of a small object which is subsequently pulled away with laser tweezers [9]. One can even create tension inside a vesicle by polymerizing a stiff rod inside it [10], whereupon a cylindrical structure modulated with pearls can emerge [11].

Recently Moses and Bar-Ziv have introduced a new class of experiments in which laser tweezers act directly on single lipid bilayers [12]. It is already perhaps surprising that there should be any action at all, since the optical absorption of a single bilayer is so small. As we will recall in Sect. 3 below, we argued in 13 that the effect of the tweezers is to induce a tension $\Sigma$ in the membrane proportional to the laser power. Our argument relied only on basic electrodynamics, and while it does not give the precise value of the constant of proportionality, it does imply that the effect of the laser is extremely simple; unlike other more mechanical probes, the possibility of complicated parasitic effects seems minimal. More importantly, laser tweezers provide for the first time the fast, delicate, and highly localized probe needed to understand membrane dynamics in detail. For example, while beaded tubes have been seen in some of the other techniques mentioned above (see Sect. 2), a unique feature of the tweezer experiment is the controlled ability to excite a "small-amplitude" (quasilinear) peristaltic modulation.

1 Experiments in which a biological intervention is introduced (for example, mutating a single protein) are comparatively abundant [3]. 
We recall the basic results of [12] in Sect. 2 below. The most intriguing phenomenon for us was the fact that when laser tweezers are applied to previously stable long cylindrical vesicles, they excite an instability ending with a "string of pearls" state. The initial wavelength of the instability is $\lambda=2 \pi R_{0} / k$, where $R_{0}$ is the initial cylinder radius and the dimensionless wavenumber is typically $k=0.8$. Remarkably, a purely local excitation due to the laser trap creates (in a certain regime) a uniformly modulated state, which invades the initial cylindrical region at a roughly constant velocity of about $v_{f} \sim 30 \mu \mathrm{m} / \mathrm{sec}$. This is not the sort of behavior we usually associate with pulling a stretchy object in a viscous fluid, and so we have a very interesting dynamical system.

The pearling phenomenon bears a superficial resemblance to the instability of a cylindrical column of water in air, studied in the classical works of Plateau and Rayleigh 14] 15]. In this situation surface tension destabilizes the cylinder, since the same volume per unit length can be contained with less surface area as a string of spherical droplets. As in other pattern-forming systems, a competition ensues: the lowest surfaceto-volume ratio comes from a small number of large droplets, but the formation of such a state is kinetically suppressed because it requires the motion of water (a conserved quantity) over long distances. For a macroscopic system the water may be treated as inviscid (high Reynolds number); Rayleigh calculated that the fastest-growing instability set in at wavenumber $k=0.70$ [15]. At micron scales, however, we are in a regime of low Reynolds number; here Rayleigh found the fastest-growing instability to be at $k=0$ [16], totally different from what is seen in [12].

Of course the situation studied in ref. [12] is not a thread of liquid surrounded by air. Long ago Tomotika considered a two-fluid model, in which a column of one viscous fluid is initially immersed in another with a certain positive surface tension [17]. One could imagine that these two fluids correspond to the interior and exterior water, so that both viscosities are equal. Specializing Tomotika's result to this case, one finds the fastest-growing mode to be at $k=0.56$. Unfortunately this simple picture is not obviously adequate for the pearling problem, nor indeed is its predicted value for $k_{\text {max }}$ ever observed; experimentally the initial wavenumber is always a bit larger, and then increases still further with time. What is missing?

For one thing, the two-fluid model neglects the presence of a material object between the inner and outer fluids, namely the membrane, except insofar as the latter somehow communicates tension induced at the trap to the entire surface. In particular Tomotika imposed continuity of the shear stress $T_{\rho z}$ across the boundary, while a material object 
located there will in general be capable of exerting tangential forces and hence causing a discontinuity in $T_{\rho z}$.

A more realistic treatment of membrane dynamics requires a three-fluid model, in which a two-dimensional fluid (the bilayer) separates two three-dimensional fluids (the water). The "tension" on the membrane can then more precisely be regarded as minus the $2 \mathrm{~d}$ pressure $\Pi$ of the intermediate fluid, which in turn emerges from the appropriate hydrodynamic equations. The key early works on membrane dynamics used models of this type to study equilibrium fluctuations [18]. In the pearling experiment the laser takes the system far from equilibrium by suddenly imposing a boundary condition on $\Pi$. In other words, the laser trap is regarded as a lipid reservoir located at $z=0$ in cylindrical coordinates, whose chemical potential suddenly jumps from zero to a negative value as the laser is switched on.

The idea that the laser effectively induces a tension $\Sigma$, and that a Rayleigh-type instability ensues, was first proposed by Moses and Bar-Ziv [12]. We substantiated this picture of the laser action in [13], showed how to estimate $\Sigma$ from the laser power, and found the fastest-growing mode $k_{\max }=0.68$, at the low end of the observed values.

The most serious lacunæ in the analysis of ref. [13] were that (a) we neglected the propagating character of the pearling instability, assuming instead that the tension was everywhere a constant, and (b) we neglected the fact that the lipid fluid is conserved. These points are related, of course. For a shape transformation to propagate outward from the trap, leaving behind a stationary shape, lipid must constantly be transported away from the moving front and into the trap. Assumption (a) gave us no possibility at all of predicting the front velocity, a readily accessible experimental quantity.

In this paper we will present a more detailed analysis addressing these and many other points. After reviewing the experimental facts in Sect. 2, we will build up a physical picture of the pearling system in Sect. 3. In particular we will show that assumption (b) above is a good approximation during the time regime of the experiment, so that pearling is essentially the invasion of a saturating front into a uniform linearly-unstable state. To get to the point as quickly as possible we will construct in Sect. 4 a very simple form of the three-fluid model which captures most of the essential physics without lengthy formulas. We then analyze the front propagation in Sect 5 using the marginal stability criterion (or

2 Actually we quoted $k_{\max }=0.65$ due to an error which we will correct in eqn. 4.2 below. 
"MSC") [19]20] and show how it gives qualitatively correct results for $v_{f}$ and $k$ when applied to our simple model. As we will recall, the MSC allows one to compute the front velocity using only the linearized dynamics.

To get quantitatively accurate predictions we will construct a more realistic model in Sect. 6. The reader may wish to skip this rather technical section. In it we first solve the hydrodynamics exactly, then resolve the bilayer into its two monolayers, to get a four-fluid model. The motivation for this is that dynamical friction between the two leaves of the bilayer has been shown to be quite significant for a related class of membrane problems 21] 22] [23], though as we will see the actual effect on our answers will be slight. Again using the MSC, we first find that the initial wavenumber is $k_{0}=0.80$, which agrees with experiment somewhat better than the result of [13]. In particular the initial wavenumber is insensitive to changes in the laser power or tubule radius, as observed. Secondly we get a front velocity $v_{f}$ equal to $0.06 \Sigma / \eta$ where $\eta$ is the viscosity of water, another prediction in rough agreement with experiment. While this prediction isn't precise due to our imprecise knowledge of $\Sigma$, it does explain why the magnitude of $v_{f}$ is observed to be so much smaller than the natural velocity scale $\Sigma / \eta$. Moreover, the linear dependence of the initial front velocity on the laser power should be verifiable in the future.

Since the MSC uses only the linearized equations it must assume, rather than proving, the existence of a uniformly-propagating front. One could take the phenomenological attitude that such behavior is observed experimentally, but this and other qualitative facts should emerge from the solution of the full nonlinear equations. In Sect. 7 we introduce a third model, with most of the simplifications of the first, simple model (Sect. 4) but retaining the nonlinear structure of the elasticity. We show the results of numerical studies that indicate that it indeed supports propagating front solutions, and that it produces stationary pearled structures like those seen in experiment. More details will appear elsewhere.

Appendix A contains a glossary of symbols used for physical constants, and their values.

Granek and Olami were the first to attempt a realistic treatment of pearling without the simplifying assumptions (a), (b) above [24]. While our physical picture and conclusions are different from theirs, we are indebted to them for emphasizing these issues. We are also grateful to Moses and Bar-Ziv, who first suggested to us that marginal stability might be applicable to this problem. Finally, others have suggested that the laser could have other effects besides inducing tension, for example effectively inducing a bilayer asymmetry (spontaneous curvature) [24] 25]. We don't see how this could happen, and in any case we will see that no such effect is needed in order to explain the observed phenomena. 


\section{Relevant Experimental Facts}

Briefly the observed phenomena of interest to us are as follows [12]. Initial preparation of the system yields stable, nearly straight cylinders up to hundreds of microns long, anchored at both ends by large globules of lipid. Each tube consists of a single bilayer of DMPC or DGDG, with water on the inside and outside. The tubules are polydisperse, with initial radii $R_{0}$ between $0.3-5 \mu \mathrm{m}$. The high temperature used $\left(45^{\circ} \mathrm{C}\right)$ implies that the membrane is in its pure fluid state. Initially the system is somewhat flaccid, as seen from visible thermal undulations and the fact that the tubes are not quite straight.

Application of a laser spot localized to $\sim 0.3 \mu \mathrm{m}$ produces a dramatic transformation to a stationary "peristaltic" figure, i.e. a cylindrical shape with radius $r(z)$ at first varying roughly sinusoidally with distance $z$ from the trap. There is no evidence for any initial time delay between laser illumination and the onset of the instability. Greater laser power is required for larger-radius tubules, but nothing seems to depend on the length of the tube, so long as the trap is initially many radii from the ends. The shape transformation propagates outward from the laser trap, with a well-defined velocity $v_{f}$ which varies between experimental trials but is typically about $30 \mu \mathrm{m} / \mathrm{sec}$ and roughly constant for at least a couple dozen wavelengths. Remarkably, after a very short illumination the shape transformation continues to propagate after the laser is shut off, leading to a uniform, small-amplitude peristaltic shape. Longer excitation leads to the eponymous pearled state. Sometimes tubes intersect each other; in this case the shape transformation can actually cross from one tube to the other upon reaching the intersection [26].

Once formed, the peristaltic shape has a well-defined initial wavelength $\lambda$ which is uniform over many microns. The dimensionless initial wavenumber $k_{0}=2 \pi R_{0} / \lambda$ is always found in the range $0.64-1.0$, and is typically about 0.8 (ref. [12], fig. 1), whatever the initial radius $R_{0}$. After prolonged tweezing some buildup of lipid becomes visible at the point of application of the laser. As the modulation grows more pronounced, $k$ grows from $k_{0}$ to become slightly greater than 1 and deviations from a simple sinusoidal profile become pronounced. The modulated state is tense: visible thermal fluctuations are suppressed and the tube draws itself straighter than initially.

We pause to contrast the above phenomena with other related results. Deuling and Helfrich studied aged red blood cells, which had different interior and exterior fluids; in this situation it is reasonable to invoke a spontaneous curvature $c_{0}$ expressing the chemical asymmetry of the bilayer environment. In our case there is no such asymmetry; as we will 
recall in Sect. 3.1 below, the fact that each leaf of the bilayer is initially in equilibrium with a common lipid reservoir then implies that $c_{0}=0$ [27].

Evans and Rawicz, and recently Pouligny, have rapidly formed thin tethers coming from large vesicles, respectively by adjusting the internal pressure or by pulling out a small inclusion in the membrane [7] [9]. Such tethers sometimes contain pearls. Also lowertemperature experiments, where in-plane order is important, can give pearled shapes [28]. All of these experiments have in common that the formation of pearls is coterminous with the formation of the tethers themselves, and small-amplitude sinusoidal modulations are not seen. Finally, experiments in mixed surfactant systems have shown equilibrium peristaltic shapes [29]. This is presumably due to shape-composition coupling, which is known to lead to an instability towards an asymmetric bilayer [30] [31] and thence to the mechanism of [32].

\section{Physical Picture}

In Rayleigh's problem, the tension was a material parameter characterizing the airwater interface, and hence trivially constant. In our case we argued in the Introduction that tension is instead a dynamical variable, and so its spread must be self-consistently determined along with the change of shape. Our main objective in this section is to justify the assumption [12] [13] that tension initially spreads so rapidly that it effectively becomes constant and uniform as soon as the laser is turned on 3 . Thus the spread of tension is not what limits the speed of initial propagation of the shape pulse. Our arguments in this section will all be rather crude. We can only emphasize that the field of membrane dynamics is not yet fully developed, and buttress our arguments whenever possible with the observed phenomenology.

In this section we will for simplicity assume axial symmetry everywhere. We will return to this point in Sect. 6.3. Thus all scalars are functions of radius $\rho$, distance $z$ from the trap, and time $t$; all velocity vectors have vanishing azimuthal component.

3 As mentioned earlier, Granek and Olami first studied this issue 24. 


\subsection{Initial equilibrium}

We begin with a discussion of the initial equilibrium, before turning on the laser.

Initially some preparation protocol has created long cylindrical vesicles constrained to stretch between the terminal blobs and in thermal equilibrium with them. We will speculate as little as possible about the nature of the blobs. Since they are far from the illumination spot, their only role is to determine the nature of the initial equilibrium, not the subsequent fast dynamics. We will simply assume that the blobs furnish lipid reservoirs and that both leaves of the bilayer membrane are in equilibrium with these common reservoirs, with a chemical potential for exchange close to zero.

With this assumption we find that initially the membrane has tension close to zero, consistent with the initially observed thermal motion [12]. We also get that the spontaneous curvature $c_{0}=0$, since in a chemically symmetric bilayer $c_{0}$ can only arise as the difference in chemical potentials between the two constituent monolayers [27]. $c_{0}=0$ is consistent with the observed absence of any preferred initial tubule radius [12]. Nonzero spontaneous curvature can lead to equilibrium unduloid shapes [32] [33], but as we mentioned earlier these are not observed in the experiments in question. Moreover, these "Delaunay surfaces" begin with an initial wavenumber $k=1$, larger than what is observed (see Appendix B).

The physical boundary condition imposed by the terminal blobs will not matter in our analysis, but for concreteness suppose that they seal off the tubule, imposing fixed volume. This constraint then creates a physical pressure difference, with a very small negative constant pressure inside the tube $p_{0}=-\kappa / 2 R_{0}^{3}$ (and $p=0$ outside), where $\kappa$ is the usual bending modulus. $p_{0}$ balances the tendency of a cylinder to increase in diameter to reduce its bending energy. One can either solve the volume constraint explicitly and substitute into the bending energy (as in 12 [13), or else regard $p_{0}$ as a Lagrange multiplier (as in [33]) to see that then the cylindrical shape is stable to all small perturbations. This is the initial equilibrium state.

4 See Appendix E. The situation is quite different in closed systems, such as vesicles of spherical topology. Here indeed the initial preparation leads to a new parameter describing vesicles, the fixed area difference between the two monolayer leaves [6]. 


\subsection{Laser action}

Next we recall our model of the laser action from ref. [13]. When the laser comes close to the membrane, nothing happens: local heating is not important. When the laser spot touches the membrane, it pulls material in by the dielectric effect. While it is hard to calculate the exact tension so induced, we may easily estimate it as follows5: the applied laser power of $\sim 50 \mathrm{~mW}$, spread over a spot of diameter $0.3 \mu \mathrm{m}$, corresponds to an energy density in vacuum $\mathcal{E}$ of $2.3 \cdot 10^{4} \mathrm{erg}^{-3}$. Taking the dielectric contrast between water and lipid at this frequency to be of order $\delta \epsilon=0.23$ [34], we see that when a lipid molecule falls into the trap displacing water we gain an energy [35] $\sim \mathcal{E} \delta \epsilon \cdot a_{0} D$, where $a_{0}$ is the area of the molecule's head and $D$ is its total length. Taking $2 D \sim 40 \AA$, we get that each unit of bilayer area sucked into the trap yields an energy gain of $\Sigma \sim 2 \cdot 10^{-3} \mathrm{erg} \mathrm{cm}^{-2}$.

Actually this figure is surely an overestimate. If the action of the laser is really to pack more lipid into the trap, there must be an offsetting cost per unit area to fold it up or otherwise put it into a more compact configuration than a single nearly-flat bilayer. (For more details see [36].) Still, we see that the trap generates a tension well in excess of the critical value [12] $\Sigma_{\text {crit }} \sim \frac{\kappa}{R_{0}^{2}} \sim 1.2 \cdot 10^{-4} \mathrm{erg} \mathrm{cm}^{-2}$ needed to trigger shape transformations (see Sect. 6.3 below), where $\kappa \sim 0.6 \cdot 10^{-12} \mathrm{erg}$ is the bending stiffness of DMPC bilayers and we took $R_{0}=0.7 \mu \mathrm{m}$ for illustration. Let $\sigma \equiv \Sigma R_{0}{ }^{2} / \kappa$ denote the dimensionless tension; thus

$$
\sigma \equiv \Sigma R_{0}^{2} / \kappa \sim 2 \cdot 10^{1}
$$

and larger for larger tubules. Such large tensions are qualitatively corroborated by other experiments involving laser-induced expulsion of vesicles [36].

One could imagine that the details of how the bilayer gets packed into the optical trap could effectively create different boundary conditions for the chemical potential of each layer and hence induce a sort of symmetry breaking, a spontaneous curvature for the layer. For example, tiny vesicles could get pinched off preferentially from the inner layer. We will resist such speculations, as we have no theoretical or experimental evidence in favor of such a mechanism, nor will such a spontaneous curvature be needed to understand the phenomena. We will also assume that the laser creates no major disruption to the integrity of the bilayer, i.e. the membrane remains impermeable on the time scale (tenths

5 We thank R. Bruinsma for suggesting this estimate. 
of a second) in question6, and transfer of lipid from one leaf to the other ("flipflop") also remains too slow to be of interest, as in unstressed membranes [37].

Further support for our model of the origin of pearling by laser-induced tension comes from the observation that mechanical tension applied suddenly to a cylindrical vesicle (by dragging an end attached to a movable pipette) creates a pearling instability similar to the one seen with the laser [26]. Whichever excitation one uses, when it stops the tension reverts slowly to zero, thermal fluctuations resume, and the tubule relaxes back to its initially stable cylindrical shape [12].

\subsection{Initial tension propagation}

As we mentioned in the Introduction, the induced tension $\Sigma$ is properly to be regarded as a boundary condition on the pressure $\Pi$ of the lipid fluid?:

$$
\Pi(z=0, t>0)=-\Sigma
$$

The sudden introduction of a gradient of $\Pi$ causes the lipid to move toward the laser spot; this motion in turn stretches the membrane, causing the tension to spread outward. We will first argue that this initial spreading of tension is very fast, and essentially complete long before shape changes have had a chance to begin. Hence in this subsection only let us at first neglect shape changes altogether and ask what happens in a linearized analysis when we begin to pull on a cylinder of $2 \mathrm{~d}$ fluid, then later check the self-consistency of this picture. Since the shape is fixed and the water is incompressible, there can be no net flow of water across a cross-section of the tube.

As the bilayer begins to move toward the trap, it entrains the surrounding water. Focusing our attention on the interior volume, this entrainment sets up a velocity profile $v_{z}(\rho, z, t)$. For this estimate we will use Poiseuille (or "lubrication") approximation, where all gradients in $z$ are assumed much smaller than those in $\rho$. (We will also temporarily neglect the exterior fluid.) The incompressible conservation law $\nabla \cdot v=0$ then implies

6 This amounts to assuming that the volume is effectively clamped by dissolved macromolecules. It is reasonable since large laser-induced tension can pressurize vesicles with no significant loss of interior volume, even long after the laser is shut off [36]. Indeed far greater tensions than those considered here can be applied mechanically with no observed loss of interior volume nor other breakdown in the bilayer model [7].

7 The pressure of a $2 \mathrm{~d}$ fluid has dimensions of tension. 
that the radial velocity is small, $v_{\rho} \ll v_{z}$, and by the equations of motion the pressure $p$ is constant across the cross-section of the tube. Solving the remaining equation of motion $\eta \nabla^{2} v_{z}=\nabla_{z} p$ in this approximation then gives us the usual parabolic velocity profile of Poiseuille flow,

$$
v_{z}(\rho, z, t)=f(z, t) \rho^{2}-g(z, t)
$$

where $f$ is related to the pressure gradient and $g=\frac{1}{2} R_{0}^{2} f$ because there can be no net flow of water down the tube.

The membrane itself is a compressible $2 \mathrm{~d}$ fluid. Its velocity $\tilde{v}$ must match that of the water:

$$
\tilde{v}=v_{z}\left(R_{0}\right)=\frac{1}{2} R_{0}^{2} f
$$

For its equation of motion, we can neglect the $2 \mathrm{~d}$ viscosity of the lipid on these scales 22 and write the force-balance equation as

$$
\nabla_{z} \Pi(z, t)=T_{z \rho}\left(R_{0}, z, t\right)=-\left.\eta \partial_{\rho} v_{z}\right|_{R_{0}}=-2 \eta R_{0} f(z, t)
$$

where $T_{i j}$ is the $3 \mathrm{~d}$ stress tensor of water. Gradients in the velocity must in turn affect the density of lipid, by another conservation law. We write the lipid density as $\phi=\phi_{0}(1+\chi)$ where $\phi_{0}$ is the equilibrium density and $\chi$ is the areal strain; then

$$
\frac{\mathrm{d}}{\mathrm{d} t}\left[\mathrm{~d} z 2 \pi R_{0} \phi_{0}(1+\chi)\right]=-\mathrm{d} z \cdot \nabla_{z}\left[2 \pi R_{0} \tilde{v} \cdot \phi_{0}(1+\chi)\right]
$$

To close the equations we need the constitutive relation

$$
\Pi=K \chi
$$

where $K$ is the $2 \mathrm{~d}$ bulk modulus of the lipid layer.

Combining we find

$$
\frac{\partial \Pi}{\partial t}=\frac{K R_{0}}{4 \eta} \nabla_{z}^{2} \Pi
$$

whose solution $\Pi(z, t)$ indeed spreads rapidly until it is essentially equal to the boundary condition $-\Sigma$ throughout the observed tens of microns. To estimate how rapidly, we need the value of $K$. We argue in Appendix $\mathrm{C}$ that $K$ is effectively much smaller than its "bare" 
value $8 K_{0} \sim 1.4 \cdot 10^{2} \mathrm{erg} \mathrm{cm}^{-2}$; instead we will argue for $K_{\mathrm{eff}} \sim 10^{-1} \mathrm{erg} \mathrm{cm}^{-2}$. Even so, $K_{\text {eff }} \gg \Sigma$. Combined with (3.1) we get the hierarchy of scales

$$
\kappa R_{0}^{-2} \ll \Sigma \ll K_{\mathrm{eff}}
$$

which we will use repeatedly.

The large modulus means that the tension profile will rapidly outrun any front of shape transformation traveling at $v_{f} \leq \Sigma / \eta$ (in fact we will find in Sect. 6 that $v_{f}$ is much smaller than this). For technical reasons the observed region must contain the laser spot, so propagation is not observed for values of $L$ larger than about $50 \mu \mathrm{m}$. Scaling (3.4) we see that at a distance $L$ from the trap the tension approaches its saturation value in a time of order $t_{L} \sim L^{2} \eta / K_{\text {eff }} R_{0}$. Taking $L=50 \mu \mathrm{m}$ gives $t_{L} \sim 4 \cdot 10^{-2}$ sec, about one video frame, and much faster than the time $L / v_{f} \sim 2$ sec needed for the observed front propagation.

We conclude that after a very short time the tweezers create a uniform, tense, stretched state of the lipid in the region of interest. If the tension $\Sigma$ is great enough, this state will be everywhere linearly unstable to shape perturbations, just as in the Rayleigh instability. Of course it remains to show that a uniform stretched state will indeed change shape via a uniformly propagating front as assumed above.

\subsection{Front propagation}

We have just argued that initially the tension spreads rapidly, before the shape has had a chance to change. To see what happens to tension as the instability progresses, we will continue to assume that this proceeds by the propagation of a front, leaving behind a stationary modulated state. Later, this is shown to be a self-consistent picture.

The energy which drives our instability comes from delivering lipid to the trap. But where does this lipid come from? Certainly very little comes from stretching the bilayer, since we just asserted that its bulk modulus is much greater than the applied tension. Instead it comes from a shape transformation, in which the initial cylindrical shape gets replaced by something with less area (and hence fewer lipid molecules) per length. It may at first be hard to see how such a transformation could propagate at a constant velocity. After all, lipid must constantly be transported to the trap, entraining water as in the

8 Were we to use the bare value $K_{0}$, we would have to include inertial effects in the above derivation, leading to a different dispersion relation from (3.4) [22], but with the same qualitative conclusion: tension spreads more quickly than shape change. 
previous subsection and incurring viscous dissipation. Much as in Poiseuille flow through a pipe, shouldn't we expect a decrease of pressure $|\Pi|$ as the front moves away from the trap?

To address the question, consider first a case in which the cylinder gets converted at a moving front to a modulated shape, with a change of its area per unit length from $2 \pi R_{0}$ to $2 \pi R_{0}(1-\alpha)$. The front is located at $z=L$, so to advance it at a velocity $v_{f}=\mathrm{d} L / \mathrm{d} t$ lipid must flow toward the trap at velocity $\tilde{v}=\alpha v_{f}$.

The laser does work on the membrane at a rate $2 \pi R_{0} \alpha v_{f} \cdot \Sigma$. This energy is lost due to viscous dissipation both at the front, and everywhere from $z=0$ to $L$. Since the front is only a few times $R_{0}$ in length, we can estimate the former loss as $\eta v_{f}^{2} \pi R_{0}$. The latter loss will be mainly due to entrainment of water. By the remarks in the previous subsection, the entrainment of water creates a shear $\partial_{\rho} v_{z} \sim \alpha v_{f} / R_{0}$ in a volume $\pi R_{0}{ }^{2} L$, for a loss rate of $\eta \alpha^{2} v_{f}^{2} \pi L$. Comparing the total loss rate to the power input, we find that

$$
v_{f} \propto \frac{\Sigma}{\eta} \frac{2 \alpha}{\zeta+\alpha^{2} L / R_{0}}
$$

Here $\zeta$ collects various order-one factors neglected in our rough estimates. We see that indeed the front cannot propagate at a constant velocity forever. But $v_{f}$ can stay roughly constant until $L>\zeta \alpha^{-2} R_{0}$, and the regime in which this happens is characterized by the fact that drag on the membrane behind the front is negligible. (The late-time asymptotics have been studied by Granek and Olami [24].)

What is the fractional area loss $\alpha$ ? Suppose first that the modulation saturates behind the front at a small amplitude, so that its radius is (Fig. 1)

$$
r(z, t)=R_{0}(1+u(z, t))
$$

with $|u| \ll 1$. Requiring that the new shape have the same volume per unit length as the old (no global transport of fluid), we find that $\alpha$ must be $\mathcal{O}\left(u^{2}\right)$, so that $R_{0} / \alpha^{2}$ is enormous. We will argue that this case is relevant for the case of a short laser pulse, when full pearls never form at all.

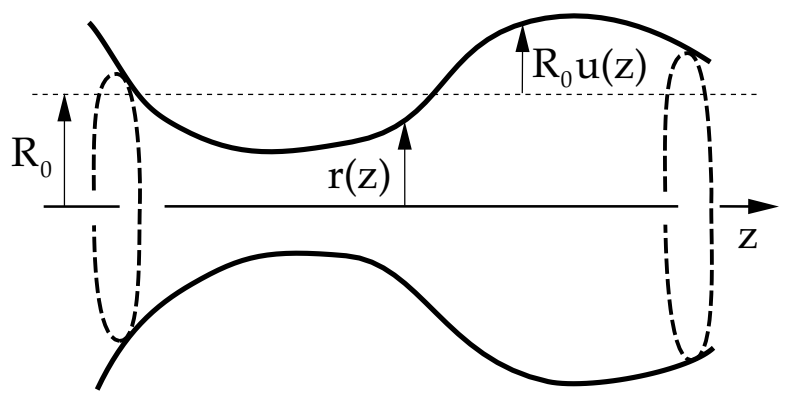

Fig. 1: Modulated cylinder. 
If on the other hand the laser is kept on for a long time, we expect the final state to be of large amplitude $u \sim 1$, so one might worry that $\alpha \sim 1$. But we know a lot about the final state: it must be a string of pearls on thin tethers. After all, neglecting bending stiffness the system minimizes area at fixed volume, leading to spherical pearls. Kinetic limitations prevent the pearls from all merging; instead we expect a periodic structure with some wavelength $\lambda$. The effect of the curvature stiffness is to prevent the tethers from shrinking to zero size. Since a thin cylinder of radius $R_{1}<R_{0}$ and length $\ell$ has curvature energy $\frac{\kappa}{2}\left(2 \pi R_{0} \ell\right)\left(\frac{1}{R_{1}}\right)^{2}, R_{1}$ will stabilize at a nonzero value. Indeed if the tension is large (as we have argued), $\Sigma / \Sigma_{\text {crit }} \gg 1$ where $\Sigma_{\text {crit }}=\kappa / R_{0}^{2}$, then we expect tethers of small radius, as seen. 9 In this case we can neglect the volume and area of the tethers altogether.

Thus volume conservation says that a string of pearls of radius $R_{2}$, separated by thin tethers of length $\ell$ (so that the wavelength $\lambda=2 R_{2}+\ell$ ), must have volume per length $\frac{4 \pi\left(R_{2}\right)^{3}}{3 \lambda}=\pi\left(R_{0}\right)^{2}$. The area per unit length is then just $4 \pi\left(R_{2}\right)^{2} / \lambda$. For the wavelengths $\lambda \sim 2 \pi R_{0}$ characteristic of the observed nonlinear regime, this corresponds to a fractional area loss $\alpha$ of just $10 \%$. In other words, the constraint of volume conservation has kept $\alpha$ small. If we carry over the estimate leading to (3.6) (even though now the amplitude is not small), we see that once again $\alpha^{-2}$ is very large and since $\zeta$ is $\mathcal{O}(1)$, we can neglect the drag along the length of the tube for the initial propagation. Any gradient of the tension must be due to the drag, and moreover we argued in Sect. 3.3 that the tension starts out uniform, so the continued smallness of the drag means that the tension stays uniform in the initial propagation regime.

The conclusion of this subsection and the previous one is that in the regime of interest we may reasonably model the effect of the laser as generating a constant uniform tension on the membrane, just as was assumed in [12] [13]. Lipid cannot literally disappear locally, but in the observed initial regime we may act as though this were the case. In other words, the pearling system behaves as if it were suddenly quenched into a uniform unstable state. This is the sort of situation in which we expect front propagation, as we recall in Sect. 5 below.

As mentioned earlier, our conviction doesn't rest solely on these crude arguments, but also on the experimental fact that indeed a front does form and propagate at constant velocity, at least initially [12]. Later in Sect. 7 we will see the front formation again in the numerical solution to a simplified model.

${ }^{9}$ In fact the minimization gives a tether radius of $\sqrt{\kappa / \Sigma}$, times some constants of order unity, which is qualitatively correct. There is thus no need to invoke spontaneous curvature to explain tether formation. 


\subsection{Other assumptions}

Here we collect other assumptions we are making.

Our axisymmetric assumption (3.7) could be generalized to allow $u(z, \varphi, t)=$ $\sum_{m} u_{m}(z, t) \mathrm{e}^{\mathrm{i} m \phi}$. The peristaltic mode is $m=0$. In Sect. 6.3 we will see that the $m \geq 2$ modes are stable. There remains the $m=1$ mode, which corresponds to wandering of the cylinder centerline. The $m=1$ mode is always soft, or critical, since it corresponds to the broken symmetry of displacement normal to the tube. As we mentioned, such wandering is indeed observed initially. However we will neglect it since (at least in the linearized analysis) it decouples from the interesting peristaltic mode.

We also assume in our hydrodynamic analysis no-slip boundary conditions between the fluid bilayer and the surrounding water. This is a standard assumption and no evidence exists to make us relax it. However, the two leaves of the bilayer can slip relative to each other, with a friction coefficient measured in the experiments of Evans and collaborators [21] [23]. We will incorporate these effects in Sect. 6 below.

\subsection{Summary}

Let us summarize this long section. We have given a qualitative, intuitive argument for a physical picture in which the laser creates a nearly uniform, tense state, both before and during the subsequent shape changes. We assumed that the latter proceeded by the propagation of a front, which then must initially move at a velocity $v_{f}$ considerably smaller than the dimensional combination $\Sigma / \eta$ (see eqn. (3.6)), as observed. We still need to show that this is the case and get a precise formula for $v_{f}$.

Since front propagation in general leaves behind a state of wavenumber $k_{0}$ different from the fastest-growing mode $k_{\max }$ [19][20], we will also need to revisit our estimate of ref. [13] to see what happens to that prediction. We do this for a simple model in Sect. 5, then for a more accurate model in Sect. 6 .

\section{Very Simple Model}

To get as quickly as possible to the heart of the matter we first develop a simple truncated model without a lot of algebra. In this tutorial model only we will make some expedient additional assumptions, each of which we will justify or improve in the sequel. The assumptions are: 
i) As in [13], we will temporarily neglect the propagating character of the modulation until Sect. 5. Instead we will just find the wavenumber of the fastest-growing Fourier mode. Our goal here is to show in the simplest possible way how tension leads to wavenumber selection.

ii) We will neglect altogether the bending stiffness of the membrane as well as all bilayer effects until Sect. 6.2. This will prove to be a good approximation if the applied tension $\Sigma$ greatly exceeds the threshold value for instability, as we have argued is the case (see eqn. (3.5)).

iii) We will neglect the finite compressibility of the membrane. This is legitimate as long as $\Sigma$ is much smaller than the effective elastic modulus $K_{\text {eff }}$ (see eqn. (3.5) and Appendix C).

iv) We will use lubrication approximation as in (3.2). This is certainly not justified, since the wavenumber $k_{\max }$ of the mode we will find is not much smaller than unity and hence $z$ derivatives are not much smaller than $\rho$ derivatives, but it will make our equations very simple. Similarly we will neglect the exterior fluid altogether.

We argued that as soon as the laser switches on, very quickly a nearly uniform tension $\Sigma$ appears. Volume conservation then implies that the pressure inside jump to a constant positive value, $p_{1}=\Sigma / R_{0}$ (the Laplace pressure of a cylinder under tension) to prevent the tube from collapsing. As the tube starts to change shape, described by the small quantity $u$ in (3.7), the Laplace pressure $\Delta p_{\text {Laplace }}(z, t)=-\Sigma \cdot 2 H$ gets new contributions from the change in the mean curvature $H$ [33]:

$$
\Delta p_{\text {Laplace }}=\frac{\Sigma}{R_{0}}\left[1-u-R_{0}^{2} \nabla_{z}^{2} u\right]+\mathcal{O}\left(u^{2}\right)
$$

Since we temporarily neglect the exterior fluid, the interior fluid must move in such a way as to give the pressure $p(r(z, t), z, t)=\Delta p_{\text {Laplace }}$, which we just computed. To linear order we again have the fluid flow (3.2), where now $f, g$ are functions of $z$ and time. We can no longer fix $g$ by demanding that the net flow of water across any cross-section vanish, since now the tube is getting fatter in some places and thinner in others. Similarly we need to modify the lipid conservation law. Generalizing (3.3) to variable radius but fixing the density gives

$$
\frac{\mathrm{d}}{\mathrm{d} t}[\mathrm{~d} z 2 \pi r(z, t)]=-\mathrm{d} z \cdot \nabla_{z}[2 \pi r(z, t) \tilde{v}]
$$


where the boundary velocity $\tilde{v}_{z}=v_{z}(r(z, t), z, t)=f r^{2}-g$. Thus linearizing in small quantities we get $\dot{u}=-\nabla_{z}\left[f R_{0}{ }^{2}-g\right]$. Similarly, conservation of the interior water gives

$$
\frac{\mathrm{d}}{\mathrm{d} t}\left[\mathrm{~d} z \pi r(z, t)^{2}\right]=-\mathrm{d} z \cdot \nabla_{z}\left[\int(2 \pi \rho \mathrm{d} \rho) v_{z}\right]
$$

or $\dot{u}=-\nabla_{z}\left[\frac{1}{4} f R_{0}^{2}-\frac{1}{2} g\right]$. Comparing (4.2) we find 10

$$
\dot{u}=\frac{1}{2} R_{0}^{2} \nabla_{z} f
$$

Since we are linearizing, let us take $u(z, t)$ to be a single Fourier mode:

$$
u_{k}(t) \cos \left(k z / R_{0}\right)
$$

The fluid equation of motion $\eta \nabla^{2} v_{z}=\nabla_{z} p$, together with (4.1) then gives (neglecting $z$ derivatives compared to $\rho$ derivatives) $4 f=-\frac{\Sigma}{\eta R_{0}} \nabla_{z}\left(1-k^{2}\right) u$, or (see Fig. 2)

$$
\dot{u}=\frac{\Sigma}{8 \eta R_{0}} k^{2}\left(1-k^{2}\right) u
$$

so that the mode with the largest growth rate $\dot{u} / u$ is at $k_{\max }=1 / \sqrt{2}$. Note that the tension needed to get an appreciable growth rate increases with tubule radius, as observed.

Note the structure of the dispersion relation (4.6); the growth rate is the product of a term $\left(\propto\left(1-k^{2}\right)\right)$ arising from the perturbation to the pressure in (4.1), while the additional overall factor of $k^{2}$ represents two spatial derivatives coming from the hydrodynamics. The first is due to the conservation of fluid volume, and the second from the usual Poiseuilleflow relation between fluid flux and pressure gradients. In the more refined hydrodynamic theory derived below the general structure is retained, in the sense that the growth rate is the product of a hydrodynamic factor and a pressure term, but the former has a more complicated wavevector dependence than $k^{2}$ as a consequence of the details of flow in cylindrical geometry.

10 Note that in [13] we mistakenly set the boundary velocity to zero instead of fixing it with (4.2)-(4.3). The formula for $k_{\max }$ below is however unaffected. 


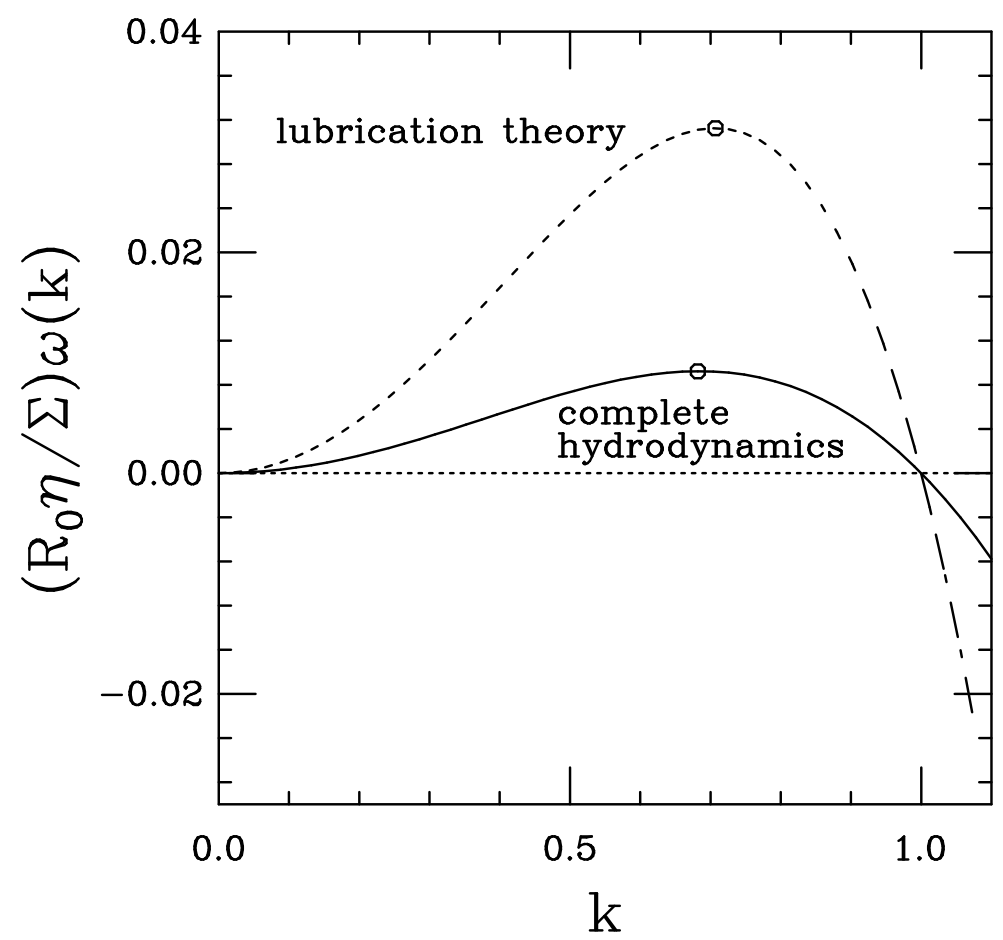

Fig. 2: Exact and lubrication approximation dispersion relations, neglecting stiffness and bilayer structure. Circles indicate fastest-growing modes.

\section{Velocity and Wavelength Selection}

We saw in our very simple model that tension destabilizes a cylinder. More generally when we incorporate bending stiffness in Sect. 6 we'll see the same thing, with a finite threshold $\Sigma_{\text {crit }}$. We now want to know what becomes of such an unstable state.

We argued in Sect. 3 that in the regime in question our problem could be regarded as a uniform, quenched unstable state: the membrane elastically transmits the laser-induced tension everywhere. The uniform unstable state changes its conformation until it saturates in one of two ways. For brief tweezing, we suppose that the laser pulls out about $1 \%$ of the area (see Appendix C), then shuts off; even when the laser stops pulling the shape transformation proceeds to an amplitude such that the projected area is reduced by about $1 \%$, i.e. $u \sim \sqrt{0.01}$. This fits with the observation of a small-amplitude peristaltic shape which continues to propagate after shutoff. For sustained tweezing, there is no such limitation, 
and the shape transformation continues until checked by the curvature energy of the thin tethers, as we argued in Sect. 3.4.

In either case a uniform quenched unstable state rolls off a potential hill to saturate nonlinearly. In this class of problems one typically finds that the instability proceeds via the propagation of a front from an initial disturbance into the unstable medium.11 The question of the precise conditions under which an equation has such a front solution are ticklish and require a more reliable knowledge of the full nonlinear equation than we possess (for example, at nonlinear orders the effects of thermal fluctuations are surely more subtle than the picture in Appendix C). However, if we take the existence of the front as an empirical fact, we can use the analysis of [20] to determine its main properties solely from the linearized dynamical equations.

The shape is governed by some nonlinear, translation-invariant equation in $u(z, t)$ We suppose a family of solutions whose envelopes are functions of $z-v t$, for various values of the velocity $v$. Only one of these solutions can correspond to the actual observed front; thus we seek the dynamically selected value $v^{*}$. The selected value must at least be stable to perturbations, in an appropriate sense which we now recall.

Suppose the front moves from negative to positive $z$ at velocity $v^{*}$ and consider the leading edge, where $u$ is still small. We need to examine the effect of small perturbations $\delta u$. In this region we may use the linearized equations, so that superposition holds and the perturbation belongs to a family $\delta u \sim \exp (\omega(q) t-q z)$ for various values of a complex wavenumber $q$, where $\omega(q)$ is the dispersion relation of the linearized equation. The envelope of this blip is then $\exp (t \operatorname{Re} \omega-z \operatorname{Re} q)$, which is itself translating at uniform velocity $v_{q}=\operatorname{Re} \omega / \operatorname{Re} q$. Of this family of perturbations, not all are dangerous to the stability of the front. A wavenumber $q$ is dangerous only if

i) $\operatorname{Re} q>0$, so that this is a leading edge solution,

ii) $\operatorname{Re} \omega>0$, so that it is growing, and iii) $v_{q} \geq v^{*}$.

Without (iii), the blip just gets left behind by the front and never gets a chance to destabilize it. If any complex $q$ meets all these conditions we will call the front unstable in its comoving frame.

11 In the present case the laser itself provides the initial disturbance; for another example see the photographs in [38]. 
Of course the assumed front solution itself corresponds at its leading edge to one particular linear solution with some $q^{*}$, and so

$$
v^{*}=\operatorname{Re} \omega_{*} / \operatorname{Re} q^{*}
$$

where $\omega_{*}=\omega\left(q^{*}\right)$. Let us now consider values of $q$ very close to $q^{*}, q=q^{*}+a+\mathrm{i} b$ for small $a, b$. Then we find

$$
v_{q}=v^{*}\left[1+\frac{a}{\operatorname{Re} \omega_{*}}\left(\operatorname{Re} \omega_{*}^{\prime}-v^{*}\right)-\frac{b}{\operatorname{Re} \omega_{*}} \operatorname{Im} \omega_{*}^{\prime}\right],
$$

where $\omega_{*}^{\prime}=\left.\frac{\mathrm{d} \omega}{\mathrm{d} q}\right|_{q^{*}}$. Since $a, b$ were arbitrary, and $q^{*}$ obeys (i), (ii) above, we can always satisfy (iii) as well unless

$$
\left.\operatorname{Im} \frac{\mathrm{d} \omega}{\mathrm{d} q}\right|_{q^{*}}=0, \quad v^{*}=\left.\operatorname{Re} \frac{\mathrm{d} \omega}{\mathrm{d} q}\right|_{q^{*}} .
$$

The three equations (5.1)-(5.2) are necessary conditions for the linear stability of a propagating front in its comoving frame [20]. We will refer to them as the "marginal stability criterion for $v^{*}$ or simply "the MSC", and use them to fix the three real variables $v^{*}, \operatorname{Re} q^{*}, \operatorname{Im} q^{*}$. There is a large literature on experimental tests of pattern selection via the MSC. Important early studies include refs. [39].

We can also use (5.1)-(5.2) to determine the selected wavenumber $q_{0}$ of the stationary pattern behind the front. In the comoving frame of the front, the saturated pattern is continuously created at the front and moves rigidly with velocity $-v^{*}$. Nodes are created in the leading edge of the front at a rate $\Omega=\operatorname{Im}\left(\omega_{*}-q^{*} v^{*}\right)$. We can interpret $\Omega$ as the flux of nodes moving toward the saturated pattern; if nodes are not created or destroyed as they pass into the non-linear region, then we must have $q_{0} v^{*}=\Omega$, or

$$
q_{0}=\operatorname{Im}\left(\omega_{*}-q^{*} v^{*}\right) / v^{*}
$$

for the wavenumber of the pattern [19]. Note that $q_{0}$ is generally different from both the fastest-growing mode $q_{\max }$ and from $q^{*}$.

Let us apply (5.3) to our very simple model. We found in Sect. 4 that $\omega(k)=$ $\frac{\Sigma}{8 \eta R_{0}} k^{2}\left(1-k^{2}\right)$, eqn. (4.6). Extending this formula to complex values of $k$ and applying (5.1)-(5.3), we find $k_{0} \equiv q_{0} R_{0}=.77$, a decay length of $3.8 R_{0}$, and $v^{*}=.20 \frac{\Sigma}{\eta} \sim$ $4 \cdot 10^{2} \mu \mathrm{m} / \mathrm{sec}$. In fact we will see in Sect. 6 that our more accurate calculation of $k_{0}$ is quite close to the very simple model, while our prediction for $v^{*}$ will be much smaller. 
The selected wavenumber $k_{0}$ is purely geometrical since we can form no length scale from tension and viscosity; in particular $k_{0}$ is independent of laser power.

Of course the very simple model is still rather crude, even with the refinement we just made. We will upgrade it to a more accurate model in Sect. 6. Since the algebra is a bit involved, the reader may want to skip this section and pass directly to the simulation results in Sect. 7.

\section{Complete Linear Model}

Now that we have some confidence from our very simple model, we need to address some of the oversimplifications listed in Sect. 4. First we will replace the lubrication approximation with the exact solution to the Navier-Stokes equation. Then we resolve the bilayer structure of the membrane to get the four-fluid model mentioned in the Introduction.

\subsection{Hydrodynamics: Exact linear theory}

Now we go beyond the lubrication theory and use the full linearized hydrodynamic equations for the interior and exterior fluids. We begin with Stokes's observation that the axial symmetry we have assumed makes our problem effectively two-dimensional. This, along with the incompressibility of the water,

$$
\frac{\partial v_{\rho}}{\partial \rho}+\frac{v_{\rho}}{\rho}+\frac{\partial v_{z}}{\partial z}=0
$$

allows us to write the interior and exterior water velocity in terms of the cylindrical stream function $\psi$ :

$$
v_{\rho}=\frac{1}{\rho} \frac{\partial \psi}{\partial z}, \quad v_{z}=-\frac{1}{\rho} \frac{\partial \psi}{\partial \rho}
$$

The Navier-Stokes equations are now two equations in two unknowns, $\psi$ and the pressure

$p$. We eliminate the latter by differentiating $\eta \nabla^{2} \vec{v}_{\rho}=\partial_{\rho} p$ with respect to $z$, differentiating $\eta \nabla^{2} v_{z}=\partial_{z} p$ with respect to $\rho$, and subtracting the two resulting equations to obtain

$$
\left(\nabla^{2}-\frac{2}{\rho} \frac{\partial}{\partial \rho}\right)^{2} \psi=0
$$

In Appendix $\mathrm{D}$ we find the stream function $\psi$ required by a single mode of shape distortion $u(t) \exp i k z / R_{0}$, enforcing the no-slip boundary condition and lipid conservation. This yields $v$ and $p$ in terms of $\dot{u}$, and ultimately the stress tensor $T_{\rho \rho}^{+}$inside and outside 
of the tube which determines the normal force balance equation to relate $u$ and $\dot{u}$, i.e. to determine the growth rate $\dot{u} / u=\omega(k)$. As mentioned in the Introduction, our boundary conditions differ from [17] and [24], where the shear stress $T_{z \rho}$ is taken to be continuous across the surface of the cylinder. This boundary condition is appropriate for two viscous fluids that meet at an interface [17]; in our case, however, there is a material object at the interface that supplies whatever forces are necessary to ensure lipid conservation and the no-slip condition. 12

The results for the growth rate have the form that generalizes (4.6) by the introduction of a more complicated "dynamical factor" $\Lambda(k)$,

$$
\omega(k)=\frac{\Sigma}{R_{0} \eta} \Lambda(k)\left(1-k^{2}\right)
$$

with $\Lambda(k)$ given by eqn. D.9 of Appendix D. Fig. 3 compares this function with the lubrication approximation $\Lambda_{\mathrm{lub}}=k^{2} / 8$. Our dynamical factor $\Lambda(k)$ differs from the one given in 13 due to the correction mentioned below eqn. (4.2); however the $\Lambda(k)$ of Fig. 3 leads to a $k_{\max }$ of .68 (see Fig. 2), very close to the value in [13].

We see that for all wavenumbers the exact dynamical factor lies below that given by lubrication theory. This implies slower growth of instabilities given the same material parameters $\Sigma, \kappa$, and $\eta$ (see Fig. 2). This increased drag relative to the lubrication approximation is sensible; the lubrication model neglects some components of the velocity gradients and hence underestimates the rate of viscous dissipation.

Inserting the growth rate (6.4) into the MSC (5.1)-(5.2) and solving the equations numerically yields $k_{0}=.75$ and $v^{*}=.06 \Sigma / \eta$. We note that the front velocity is indeed much slower than $\Sigma / \eta$, consistent with our discussion of (3.6).

We see that taking the propagating character of the instability into account has slightly improved the agreement with the experimentally observed $k_{0}$. Using our estimated value for $\sigma$, eqn. (3.1), we also get the front velocity $v_{f} \sim 100 \mu \mathrm{m} / \mathrm{sec}$. Since our value for $\Sigma$ is an overestimate we get $v_{f}$ squarely in the observed range.

12 In Sect. 6.3 we also address the effect of the two-dimensional membrane viscosity and show that it is dominated by the traction of the water. 


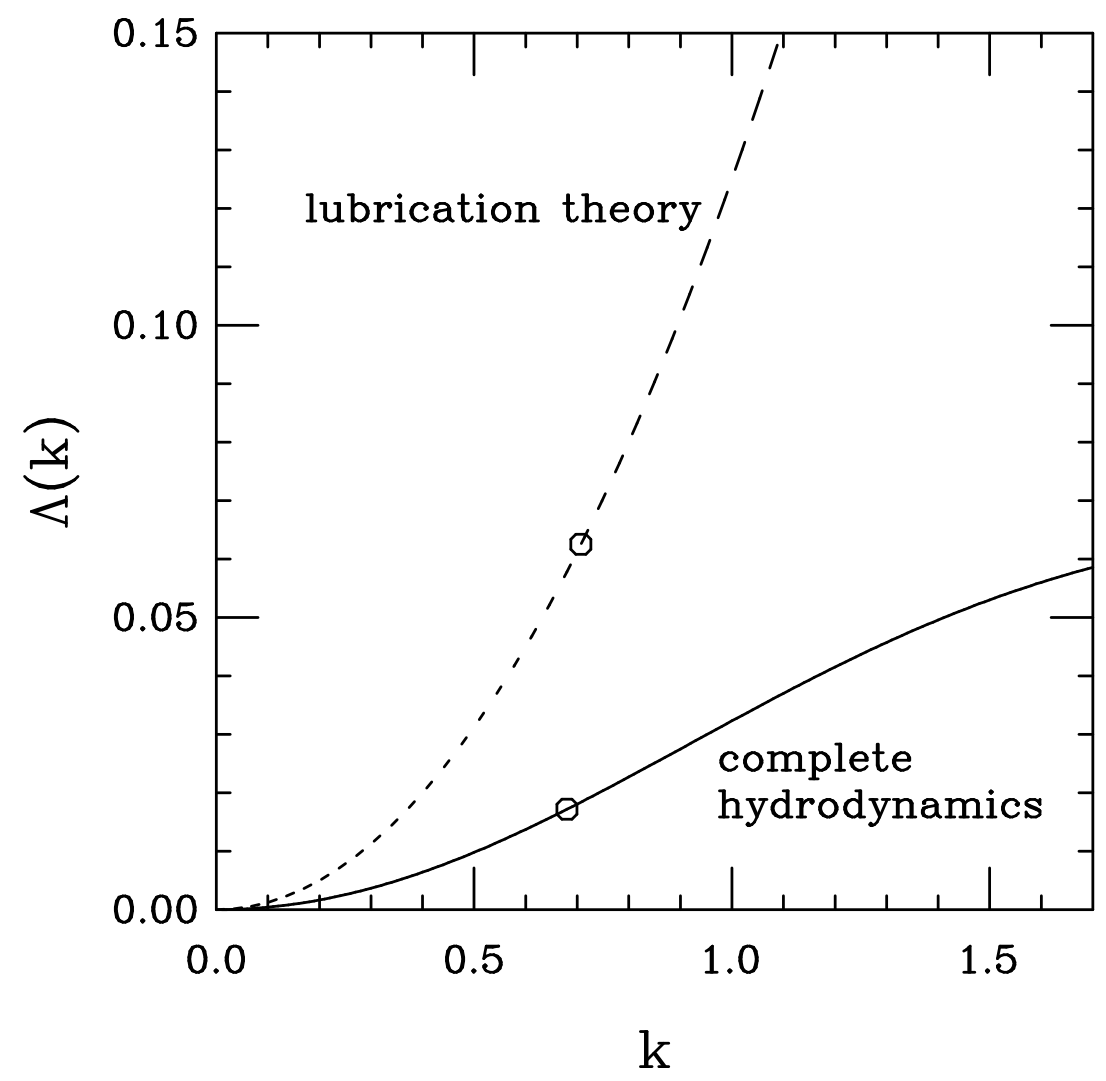

Fig. 3: Dynamical factor as a function of $k$, with fastest-growing modes indicated.

\subsection{Bilayer model}

We now need a more realistic model of the bilayer. For one thing, we have so far neglected bending stiffness, which as we saw is eventually crucial to stabilize the tethers between pearls. But stiffness effects are comparable to effects involving the differential stretching of the two leaves of the bilayer [27] [6], so we must include these as well. This means introducing more continuum degrees of freedom, the densities $\phi^{ \pm}$of lipid molecules per unit area in each monolayer.

In fact one must sometimes retain $\phi^{ \pm}$even in equilibrium problems, for example in closed systems where the numbers of molecules are separately constrained. This situation leads to the "area-difference elasticity" model studied for example in ref. [6]. We can simply quote their intermediate formula for the elastic energy:

$$
F\left[H, \phi^{ \pm}\right]=\int \mathrm{d} S\left[\frac{\kappa}{2}(2 H)^{2}+\frac{K_{0}}{4} \sum_{ \pm}\left(\frac{\phi^{ \pm}}{\phi_{0}}-1\right)^{2}+\Sigma\right]
$$


(For completeness we rederive this formula in Appendix E.) Here $\kappa$ and $K_{0}$ are the bilayer stiffness and compression moduli, and $\mathrm{d} S$ is the area element of the midplane. $H$ is the mean curvature of the bilayer midplane; in our convention $H=-1 / 2 R_{0}$ for a cylinder. We measure the lipid density $\phi^{+}$at the neutral surface of the outer monolayer, assumed a distance $d$ away from the bilayer midplane, and similarly $\phi^{-}$. See Fig. 4 . Thus the parameters of the model are $\kappa, K_{0}, d$, and the full bilayer thickness $2 D$ (see Appendix A). Since $D$ and $d$ are much smaller than any other length scale in the problem, we will work to leading nontrivial order in them, as indeed we have already done to get (6.5).

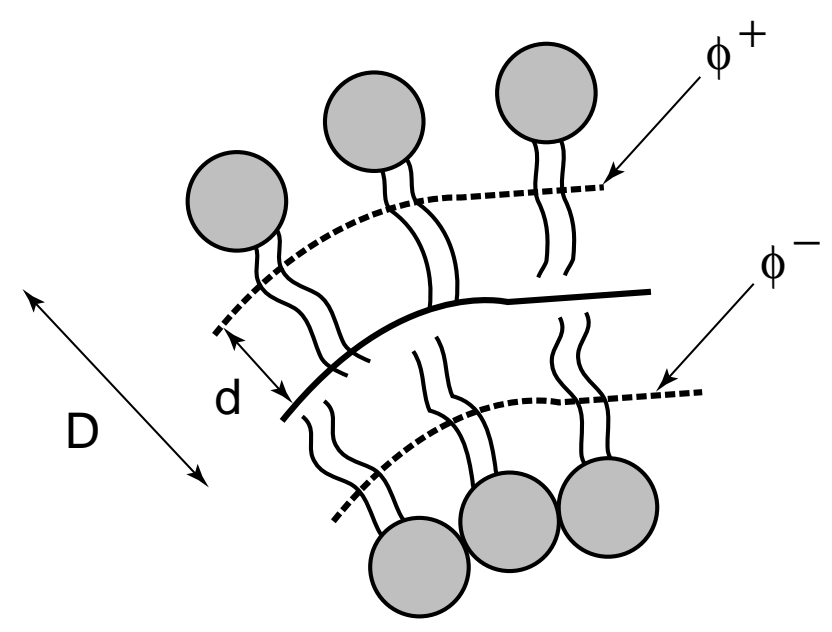

Fig. 4: Schematic of the bilayer showing various variables.

We begin in equilibrium at zero tension. Here the densities $\phi^{ \pm}$take their preferred value $\phi_{0}$. Actually we will find it useful to recast our equations in terms of the densities referred not to the monolayer neutral surfaces, but to the bilayer midplane. If we imagine each molecule casting its shadow on the midplane, the new density variables $\chi^{ \pm}$are the excess density fluctuations of those shadows 13 , relative to the equilibrium densities $\phi_{0}^{ \pm}$ projected to the midplane of the unperturbed surface. Due to the curvature, $\phi_{0}^{ \pm}$are no longer equal, but instead $\phi_{0}^{ \pm}=\phi_{0}\left(1 \mp 2 H_{0} d\right)$, where $H_{0}$ is the equilibrium curvature. Thus we have $1+\chi^{ \pm}=\phi^{ \pm}(1 \mp 2 H d) / \phi_{0}^{ \pm}$, or

$$
\phi^{ \pm} / \phi_{0}=1+\chi^{ \pm} \pm 2(\delta H) d
$$

where $\delta H=H-H_{0}$ is the change of curvature. Substituting in (6.5) above yields [13]

$$
F\left[H, \chi^{+}, \chi^{-}\right]=\int \mathrm{d} S\left\{\frac{\kappa}{2}(2 H)^{2}+\frac{K_{0}}{4}\left[\left(\chi^{+}+2(\delta H) d\right)^{2}+\left(\chi^{-}-2(\delta H) d\right)^{2}\right]+\Sigma\right\},
$$

${ }^{13}$ In [13] we called these variables $\rho^{ \pm}$. 
Were we to consider fluctuations about flat membranes, we would set $H_{0}=0$; then (6.7) reduces to the formula in [22].

Later we will want to rephrase (6.7) in terms of the mean density and density difference, and rescale:

$$
\bar{\chi}=\left(R_{0} / d\right)\left(\chi^{+}+\chi^{-}\right), \quad \hat{\chi}=\left(R_{0} / d\right)\left(\chi^{+}-\chi^{-}\right)
$$

We argued in Sect. 3 that the mean density adjusts quickly to its preferred value $\bar{\chi}=0$, since only hydrodynamic drag obstructs this, and initially it is not effective. We cannot be quite so sure about density difference, however. The two monolayers are like a pair of polymer brushes in contact, and so the friction coefficient between the two layers can be (and is) enormous [40] [23]. Leaving $\hat{\chi}$ free, we then finally obtain the elastic energy

$$
\begin{aligned}
F[H, \hat{\chi}]=\int \mathrm{d} S\{ & \frac{\kappa}{2}(2 H)^{2}+\frac{K_{0} d^{2}}{2}\left(2 H-2 H_{0}\right)^{2} \\
& \left.+\frac{K_{0}}{2}\left(\left(\frac{d}{2 R_{0}}\right)^{2} \hat{\chi}^{2}+\frac{2 d^{2}}{R_{0}}\left(H-H_{0}\right) \hat{\chi}\right)+\Sigma\right\} .
\end{aligned}
$$

To gain a physical feeling for (6.9), consider two limiting cases. If the interlayer friction is small (or time scales long), we can minimize (6.9) with respect to $\hat{\chi}$ also, and recover once again the Canham-Helfrich model. If the friction is very large (or time scales very short) then $\hat{\chi}$ cannot change at all from its initial value of zero, and we drop the third term of (6.9). For flat membranes, $H_{0}=0$ and the first two terms combine to give the effective increase of stiffness found in [22].

\subsection{Cylinder perturbations}

Let us instead specialize (6.9) to the case of a cylindrical initial state and a small sinusoidal perturbation of $u$ and $\chi$ of wavenumber $k$. Working to quadratic order in fluctuations of shape and density difference one has 33]

$$
\begin{aligned}
H= & \frac{1}{2} R_{0}{ }^{-1}\left[-1+u+R_{0}^{2} \nabla^{2} u-u^{2}\right] \\
& +\frac{1}{2} R_{0}\left[-\frac{1}{2}(\nabla u)^{2}-2 u \nabla^{2} u+\left(\nabla_{z} u\right)^{2}+2 u \nabla_{z}^{2} u\right] \\
\mathrm{d} S= & \left(R_{0} \mathrm{~d} z \mathrm{~d} \varphi\right)\left(1+u+\frac{1}{2} R_{0}^{2}(\nabla u)^{2}\right)
\end{aligned}
$$


In these formulas $\nabla=\left(\nabla_{z}, R_{0}{ }^{-1} \nabla_{\varphi}\right)$ is the gradient on the unperturbed cylinder. Substituting gives 14

$$
F[u, \hat{\chi}]=\frac{\kappa}{2 R_{0}^{2}} \int 2 \pi R_{0} \mathrm{~d} z\left[\text { const. }+(2 \sigma-1) u+\mathcal{Q}\left(-R_{0}{ }^{2} \nabla^{2}\right) u^{2}+\frac{\beta}{4} \hat{\chi}^{2}+\beta\left(1-k^{2}\right) \hat{\chi} u\right]
$$

where $\beta \equiv K_{0} d^{2} / \kappa$ and

$$
\mathcal{Q}(x) \equiv 1+\left(\sigma-\frac{1}{2}\right) x+x^{2}+\beta(1-x)^{2}
$$

This is our final formula for the elastic energy. As we increase the dimensionless tension $\sigma$ (eqn. (3.1) ) from zero, eventually at some $\sigma_{\text {crit }}$ this quadratic form acquires a negative eigenvalue and the system becomes unstable. (In Sect. 4 we dropped all but the $\sigma$ terms.)

We note in passing that for non-axisymmetric shapes, $u(z, \varphi, t)=u_{k m} \cdot \cos \left(k z / R_{0}+\right.$ $m \varphi$ ), for $m>1$ the polynomial (6.11) remains stable until larger values of $\sigma$ than for the peristaltic $m=0$ modes.

Having found the energy cost $F$ for small distortions in shape and relative density difference (6.10)-6.11), we now need the dynamical equations for the two monolayers, the $2 \mathrm{~d}$ fluids of our four-fluid model. These equations are the equations of tangential force balance. Each monolayer feels forces due to inhomogeneities in density, the traction $T_{\rho z}^{ \pm}$of the $3 \mathrm{~d}$ water, $2 \mathrm{~d}$ viscous forces, and the friction between the two leaves of the bilayer [22]:

$$
-\partial_{z} \frac{\delta F}{\delta \chi^{ \pm}} \pm T_{\rho z}^{ \pm}+\mu \partial_{z}^{2} \tilde{v}^{ \pm} \mp b\left(\tilde{v}^{+}-\tilde{v}^{-}\right)=0
$$

Here $\mu$ is the $2 \mathrm{~d}$ lipid viscosity; we have written the frictional force per unit area as $b\left(\tilde{v}^{+}-\tilde{v}^{-}\right)$where $b$ is a constant and $\tilde{v}^{ \pm}$are the tangential layer velocities [21] 223] [22]. Although the change in $\bar{\chi}$ may be neglected just as in Sect. 6.2, now we must keep elastic terms corresponding to changes in $\hat{\chi}$, since these are in principle of the same order as the friction term. The second and third terms of (6.12) are negligible compared to the first and the last. Indeed, since $\tilde{v}^{ \pm} \sim \dot{u} / k$, and $\tilde{v}^{+}-\tilde{v}^{-} \sim \frac{d}{R_{0}} \dot{u} / k$, the last three terms are

14 This formula differs from the one in [13] because we no longer need to replace the linear terms using the ansatz $u=-\left(u_{k}\right)^{2}+2 u_{k} \cos \left(k z / R_{0}\right)$. Instead we will see how the terms linear in $u$ determine the constant part of the pressure difference. This rearrangement of the algebra has no effect on the answers, but the presentation here emphasizes that global volume conservation is not needed and hence the detailed structure of the terminal blobs is unimportant. 
of magnitude $\eta \dot{u}, \frac{\mu}{R_{0}} \dot{u}, b d \dot{u}$ respectively, so that on micron scales the last term dominates (see Appendix A).

To get an equation for $\partial_{t} \hat{\chi}$, we use the difference in lipid conservation laws $\partial_{z}\left(\tilde{v}^{+}-\right.$ $\left.\tilde{v}^{-}\right)=-\frac{d}{R_{0}} \partial_{t} \hat{\chi}$ to write the difference of the dominant terms of (6.12) as

$$
\partial_{z}^{2}\left(\frac{\delta F}{\delta \chi^{+}}-\frac{\delta F}{\delta \chi^{-}}\right)=-2 b \partial_{z}\left(\tilde{v}^{+}-\tilde{v}^{-}\right)
$$

or

$$
\partial_{t} \hat{\chi}=-\frac{k^{2}}{b d^{2}} \frac{\delta F}{\delta \hat{\chi}}
$$

Next we need the equation of normal force balance analogous to D.7. The Laplace force law, eqn. (4.1), needs to be modified to account for bending stiffness. To find the right expression we have only to recall the origin of the Laplace law itself: the pressure jump is $\delta F / \delta V$, the change in the elastic free energy due to a small change in shape which changes the volume by $\delta V$. To get (4.1) we used $\frac{\delta F}{\delta V}=\frac{\delta F / \delta u}{\delta V / \delta u}$ with $\left.\frac{\delta F}{\delta u}=R_{0} \Sigma\left(1-R_{0}{ }^{2} \nabla^{2} u\right)+\cdots\right)$ and $\frac{\delta F}{\delta V}=R_{0}{ }^{2}(1+u+\cdots)$. Now we simply use the full elastic energy (6.10)-(6.11).

We may neglect the difference in monolayer velocities for the purposes of computing the hydrodynamic factor $\Lambda(k)$. Once again, this difference is suppressed by $d / R_{0}$ relative to the central flow velocity and therefore does not significantly modify the energy dissipated in the $3 \mathrm{~d}$ fluids. Thus we again have $\dot{u}$ given by $-\Lambda(k) / \eta$ times the nonconstant part of the pressure jump, just as in D.8. Combining with (6.13), our linearized hydrodynamic equations are

$$
\frac{\partial}{\partial t}\left(\begin{array}{l}
u \\
\hat{\chi}
\end{array}\right)=-\frac{\kappa}{R_{0}^{3} \eta}\left(\begin{array}{cc}
\Lambda(k) & \\
& \epsilon k^{2}
\end{array}\right)\left(\begin{array}{cc}
\mathcal{Q}\left(k^{2}\right)+\frac{1}{2}-\sigma & \frac{1}{2} \beta\left(1-k^{2}\right) \\
\frac{1}{2} \beta\left(1-k^{2}\right) & \beta / 4
\end{array}\right)\left(\begin{array}{l}
u \\
\hat{\chi}
\end{array}\right)
$$

where $\epsilon \equiv R_{0} \eta / b d^{2}$ is a measure of the importance of interlayer friction. The desired dispersion relation is then given by the positive eigenvalue, if any, of this linear problem, i.e. $\omega \sim\left(-180 \mathrm{sec}^{-1}\right) \cdot \lambda$, where we took a typical $R_{0}=0.7 \mu \mathrm{m}$ and $\lambda$ solves $\lambda^{2}-\lambda\left(g_{1}+\right.$ $y)+g_{0} y=0$. Here $g_{0}=\Lambda(k)\left[\frac{3}{2}-\sigma+k^{2}\left(\sigma-\frac{1}{2}\right)+k^{4}\right]$ is the growth rate with zero friction, $g_{1}=g_{0}+\Lambda(k) \beta\left(1-k^{2}\right)^{2}$ is the growth rate at infinite friction, and $y \equiv \beta \epsilon k^{2} / 4$.

Fig. 5 shows the growth rate with $\epsilon=.5, \beta=3.5$, and various values of tension. Clearly there is little effect of stiffness on the curve $\omega(k)$ when the tension is as large as its experimental value $\sigma \sim 20$ (compare with Fig. 2). For this value we read off a fastest growing mode with $k_{\max }=.68$ for $\sigma=20$, but as we argued in Sect. $5 k_{\max }$ is not the expected wavenumber. Instead inserting the above formulas for the dispersion $\omega(k)$ 


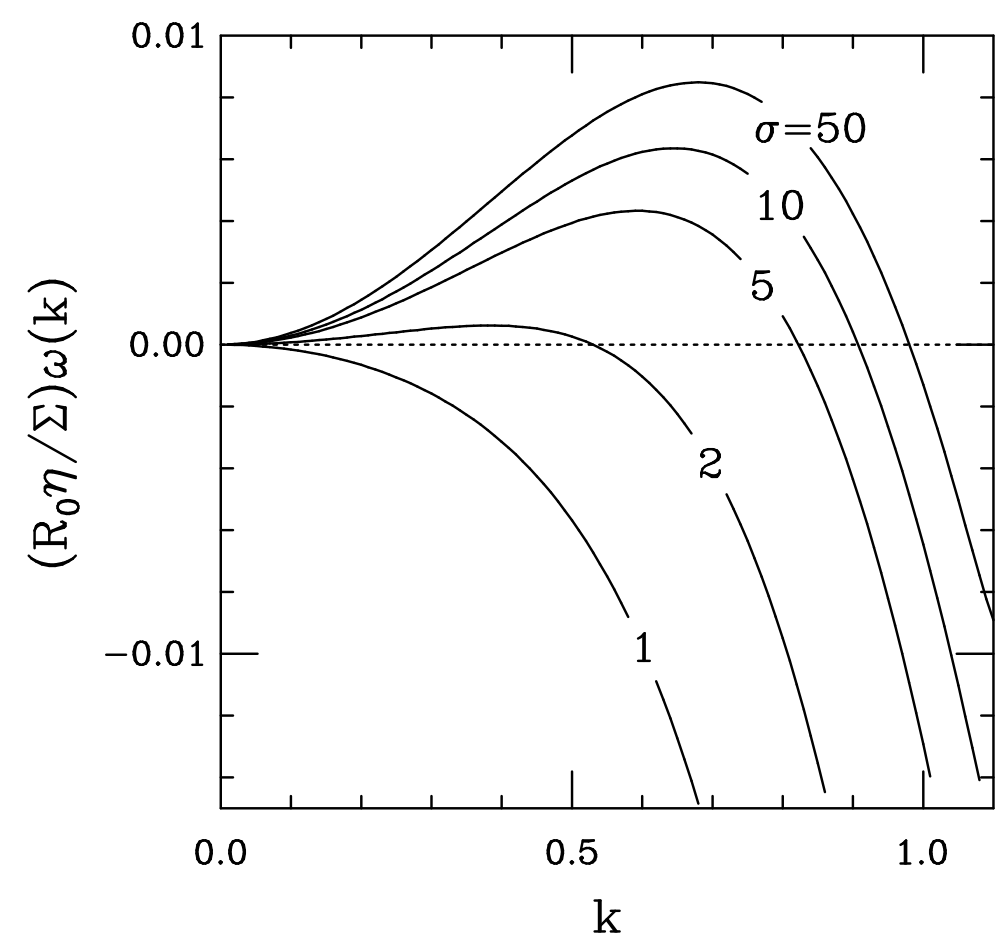

Fig. 5: Growth rate versus wavenumber in the full linear model for various values of the dimensionless tension $\sigma$. A typical experimental value is $\sigma \sim 20$.

into the equations for the MSC (5.1)-(5.2) gives $k_{0}=.80$, a decay length of $4.0 R_{0}$, and $v_{f}=.06 \frac{\Sigma}{\eta} \sim 100 \mu \mathrm{m} / \mathrm{sec}$. The perfect agreement of $k_{0}$ with Fig. 1 of [12] is fortuitous, but certainly the MSC gives reasonable values for the pattern wavenumber and front velocity, and as promised these values are not very different from those found in Sect. 6.1 since at large tension both the stiffness and the layer friction are unimportant (see eqn. (3.5)).

\section{Model for the Nonlinear Regime}

We turn finally to a model for the development of pearls beyond the linear instability. A model based on the exact hydrodynamics coupled to the elastic forces presents a rather formidable computational challenge. It is therefore of interest to define a simplified model which nevertheless maintains certain central features of the full problem. Chief among these are (i) conservation of fluid volume within the vesicle, (ii) the monotonic decrease in 
energy associated with low Reynolds number flow, and (iii) the fully nonlinear structure of the elastic energy needed to arrive at a true minimum of the energy functional.

The simplest model consistent with these constraints utilizes the lubrication approximation, thus yielding a local evolution equation for the vesicle radius $r(z, t)$. One goal of this investigation is to determine whether propagating fronts of constant velocity are indeed possible within such a model. Their existence would be strongly suggestive that such fronts are supported by the full hydrodynamics. A second goal is to test the validity of the marginal stability criterion. While the assumptions of lubrication theory may not be quantitatively correct in comparison with experiment, the issue at hand is one of internal consistency; within the assumptions of lubrication theory, how accurate is the MSC?

In the model, the diffusion of tension is ignored, so that $\Sigma$ is assumed to be uniform along the vesicle. The bilayer bending stiffness is included to stabilize pearls against pinching off, but the small effects of membrane compressibility and bilayer friction are omitted. Rather than introducing the optical trap explicitly, we consider an initial condition of uniform tension with a localized shape perturbation. We justified this approach at length in Sect. 3 .

We first need some formulas for the elastic force beyond linear approximation. The pressure difference across the membrane is the change in energy with respect to volume, so now our generalization of (4.1) becomes

$$
\Delta p=\frac{\delta F / \delta r}{\delta V / \delta r}=\frac{\delta F}{\delta V}=2\left\{-\Sigma H+\kappa\left(\hat{\nabla}^{2} H+2 H^{3}-2 H K\right)\right\}
$$

where $\hat{\nabla}^{2}$ is the covariant laplacian on the curved surface, and $K$ is the Gauss curvature. Axially symmetric shapes described by a radius $r(z)$ (Fig. 1) have mean and Gaussian curvatures given by

$$
\begin{aligned}
& H=\frac{1}{2}\left(\frac{r_{z z}}{\left(1+r_{z}^{2}\right)^{3 / 2}}-\frac{1}{r\left(1+r_{z}^{2}\right)^{1 / 2}}\right) \\
& K=-\frac{r_{z z}}{r\left(1+r_{z}^{2}\right)^{2}} .
\end{aligned}
$$

Likewise, for these shapes the determinant $g$ of the metric tensor and the covariant laplacian are

$$
\begin{aligned}
g & =r \sqrt{1+r_{z}^{2}} \\
\hat{\nabla}^{2} & =\frac{1}{r \sqrt{1+r_{z}^{2}}} \frac{\partial}{\partial z} \frac{r}{\sqrt{1+r_{z}^{2}}} \frac{\partial}{\partial z}
\end{aligned}
$$


For uniform tubular states of radius $R_{0}$ we see from (7.1) that the balance of forces yields an equilibrium radius $R_{0}=(\kappa / 2 \Sigma)^{1 / 2}$, as discussed earlier in the context of the thin "tethers" between pearls.

Recalling the derivation of the lubrication theory results in Sect. 4, in particular Eq. (4.3) , we know that the equation of motion for the radius $r(z, t)$ should have the form of a local conservation law,

$$
\frac{\partial}{\partial t} \pi r^{2}=-\frac{\partial J}{\partial z}
$$

where $J$ is the axial current. For this we appeal to our previous (linearized) result on lubrication theory that relates $J$ to the gradient of pressure,

$$
J=-\frac{\pi}{4 \eta} r^{4} \frac{\partial P}{\partial z} .
$$

Recall that the factor of 4 in the denominator differs from the usual Poiseuille result due to the imposition here of lipid conservation. The pressure is just that determined by the energy functional, eqn. (7.1). Combining Eqs. (7.2), (7.3), and (7.1), and rescaling both $r$ and $z$ with the unperturbed radius $R_{0}$ and introducing the rescaled time $\tau=\left(\eta R_{0}^{3} / 4 \kappa\right) t$ we obtain the partial differential equation

$$
r \frac{\partial r}{\partial \tau}=-\frac{\partial}{\partial z}\left[r^{4} \frac{\partial}{\partial z}\left(-\sigma H+\hat{\nabla}^{2} H+2 H^{3}-2 H K\right)\right],
$$

where $\sigma$ is again the rescaled tension (3.1).

It is easily demonstrated that this is a gradient flow, for the time derivative of the functional $F$ is

$$
\begin{aligned}
\frac{\mathrm{d} F}{\mathrm{~d} \tau} & =\int \mathrm{d} z \frac{\delta F}{\delta r} \frac{\partial r}{\partial \tau}=\int \mathrm{d} z \frac{\delta F}{\delta r} \frac{1}{r} \partial_{z}\left(r^{4} \partial_{z} \frac{1}{r} \frac{\delta F}{\delta r}\right) \\
& =-\int \mathrm{d} z r^{4} \partial_{z}\left(\frac{1}{r} \frac{\delta F}{\delta r}\right)^{2} \leq 0,
\end{aligned}
$$

where the last line follows by an integration by parts. Thus, provided $r>0$ (i.e. the interface does not pinch off), $F$ is driven strictly downhill. Furthermore, when $F$ is constant in time the functional derivative $\delta F / \delta r=0$ and the system is at an energetic extremum. Since the model (7.4) also contains the proper linear stability result of the lubrication approximation, it provides a dynamics that interpolates between the basic Rayleigh-like instability and the stationary final states, while obeying the relevant hydrodynamic conservation laws. 
The flux form of the equation of motion and the relation between pressure gradients and velocity in (7.4) are features found as well in models introduced recently for interface motion leading to topology transitions and singularities in viscous flows [41. In all of these systems, despite the simplicity of the physical ingredients, the equation of motion is highly nonlinear and of very high degree (sixth order in $z$-derivatives in the present case). These features make numerical studies quite delicate, but with care a stable and accurate algorithm may be developed. Here we report preliminary results of simulations illustrating that propagating fronts of peristalsis are supported by the model.

Fig. 6 shows the evolution of a cylindrical vesicle with $\sigma=10$ perturbed initially with a localized distortion. The computational domain has a length $16 \times 2 \pi$, and periodic boundary conditions are imposed by use of a pseudospectral algorithm. The figure clearly shows that following an initial "induction" period there is a propagating peristaltic pattern moving symmetrically outward from the initial perturbation. A nonlinear least-squares fit of the leading edge of the front at successive times to the product of a decaying exponential and a cosine function shows that the position of the front increases linearly with time over the duration of the simulation, during which it has moved about six pearl lengths. This constant velocity is in accord with our physical arguments of Sects. 3-4. We find the dimensionless wavenumber of the pattern in the leading edge of the front to be $k_{0} \approx 0.78$, and the decay length to be $\approx 5.4$. Applying the techniques of the earlier sections to the model of this section, one finds that the MSC predicts a wavenumber $k_{0}=0.77$; the decay length of 4.3 is somewhat smaller than the value observed in the numerical solution, but is still on the order of a pearl size. Thus the front is "sharp." Finally, in the rescaled units of (7.4), the front velocity at the value $\sigma=10$ is found to be $v_{f} \approx 13$, close to but somewhat smaller than the marginal stability criterion value of 17 . Work is in progress to understand the origins of these discrepancies.

In addition to these quantitative aspects of front propagation, we see that the state left behind the front indeed has the appearance of a string of pearls, with very narrow tethers between somewhat prolate ellipsoids. These are quite similar to ones seen in the experiments of Bar-Ziv and Moses. It is also interesting to note that a slight shift between the selected wavelength near the leading edge of the front and that found well behind, where the pearls are fully formed. This difference is in qualitative accord with what one finds in other more familiar examples of propagating pattern selection [19]. 


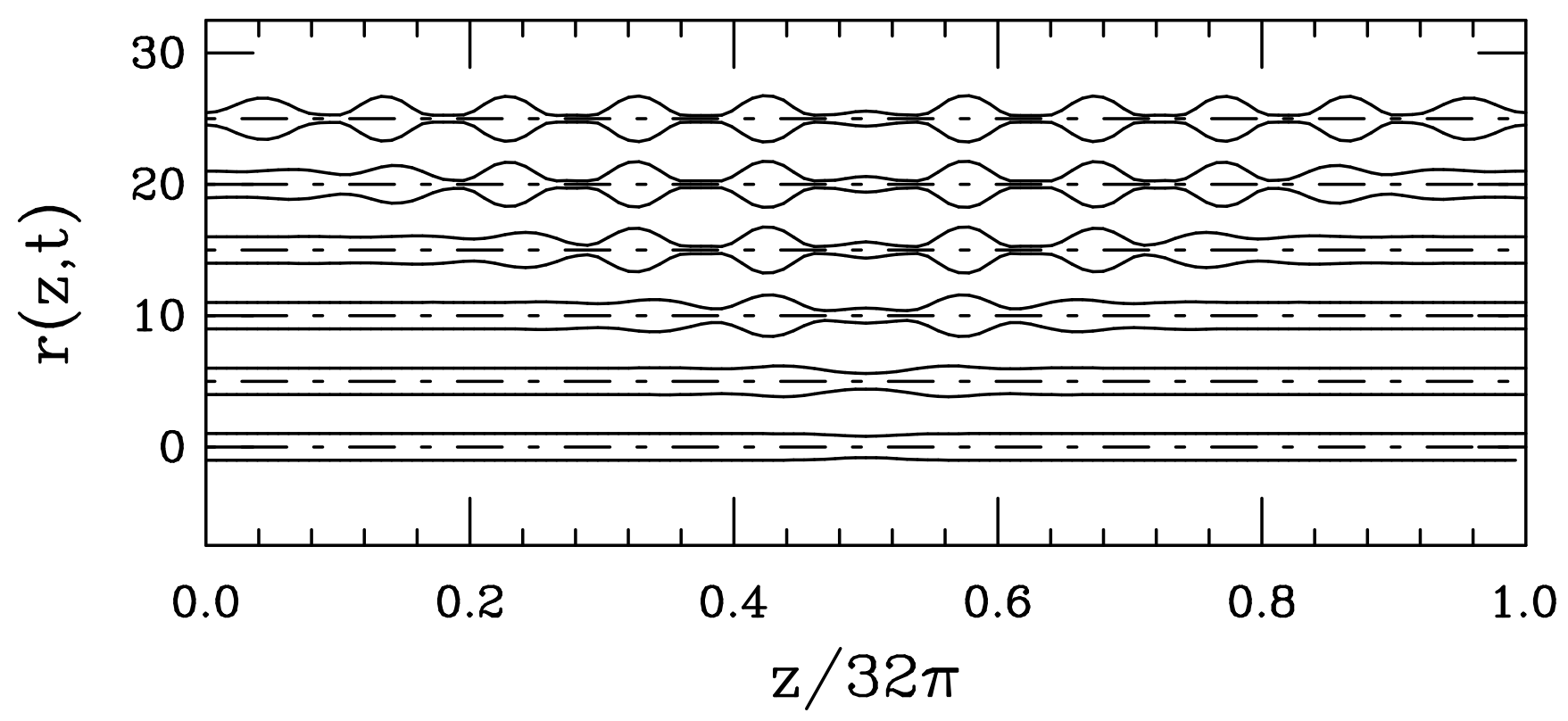

Fig. 6: Numerical solution of the lubrication equation (7.4) with $\sigma=10$, showing the formation of pearls. Time increases from bottom to top in increments of $\Delta \tau=1.0$.

\section{Conclusion}

Laser tweezers are an important new experimental tool for understanding membrane dynamics; they furnish a precisely controllable, local, physical intervention. In this paper and [13] we have proposed a simple model for the effective laser-membrane interaction and used it to explain much of the phenomenology of the pearling instability discovered in this context by Bar-Ziv and Moses.

Various aspects of the pearling phenomenon remain to be explained. For example, we have not attempted to study the migration of the pearls which develop after prolonged tweezing. What we did show was how a cylindrical vesicle under tension is unstable to states with a periodic modulation in diameter. We refined our previous prediction for the initial wavelength of this perturbation using the marginal stability criterion and predicted a selected wavenumber in good agreement with the experiments of ref. [12]. We also explained the observed front behavior and predicted a front velocity in qualitative agreement with the experiments. Along the way we showed how to incorporate the bilayer membrane structure into the dynamics. Although this structure did not significantly affect our results, it may matter in dynamics problems at lower tension and on shorter length scales. Finally, our numerical calculations have demonstrated that the lubrication approximation is rich enough to capture the essential physics of the pearling phenomena, such 
as the existence of an initial uniform front propagation velocity and the shape of the fully developed pearls. These calculations were in reasonable agreement with the predictions of the MSC, thus bolstering our faith in this approach and suggesting that the MSC can be reasonably applied to other fluid dynamics problems. One particularly relevant problem is the propagation of the ordinary Rayleigh instability along a column of fluid, as seen in recent experiments on droplet fission [42].

We are not aware of any naturally-occurring biological process to which the pearling instability literally applies. Our goal was rather to create a simple theory of membrane dynamics and test its many underlying assumptions by applying it to a well-controlled dynamical shape transformation. We expect that the approach developed in this paper can provide a starting point for understanding other phenomena of more direct biological interest, for instance the dynamics of shape instabilities during adhesion and budding.

\section{Acknowledgements}

We would like to thank R. Bruinsma, F. David, B. Fourcade, M. Goulian, R. Granek, J. Krug, R. Lipowsky, T. Lubensky, X. Michalet, S. Milner, D. Nelson, Z. Olami, V.A. Parsegian, S. Safran, and S. Svetina for innumerable discussions. We especially thank R. Bar-Ziv and E. Moses for constant help and in particular for suggesting the applicability of the MSC. PN also thanks CEA Saclay, the Weizmann Institute, and the Institute for Advanced Study, and PN and US thank ITP Santa Barbara for their hospitality and partial support, while some of this work was done. REG acknowledges support from NSF PFF Grant DMR93-50227 and the Alfred P. Sloan Foundation. PN acknowledges NSF grants PHY88-57200 and DMR95-07366, and the Donors of the Petroleum Research Fund, administered by the American Chemical Society, for the partial support of this research.

\section{Appendix A. A Compendium of Constants}

Here we list the values of various physical constants for DMPC bilayers. We take: the bilayer bending stiffness to be $\kappa=0.6 \cdot 10^{-12} \mathrm{erg}$ [43],

the bilayer thickness to be $2 D=40 \AA$ [4], 
the distance from bilayer midplane to each monolayer neutral surface to be $d \sim D / 3=12 \AA$ 40] 6],

the bare bilayer compression modulus to be $K_{0}=144 \mathrm{erg} \mathrm{cm}^{-2}$,43],

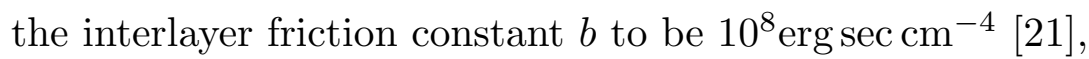

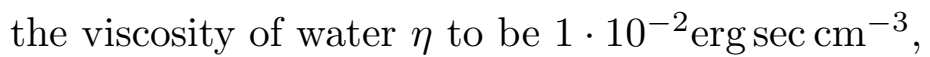

and the $2 \mathrm{~d}$ viscosity of the lipid layer to be $\mu=10^{-6} \mathrm{erg} \mathrm{sec} \mathrm{cm}^{-2}$.45.

In addition for concreteness we sometimes take:

the typical tubule radius $R_{0}=0.7 \mu \mathrm{m}$ (ref. [12], fig 1),

the typical laser power $50 \mathrm{~mW}$ [12],

and the temperature to be $k_{B} T=4.2 \cdot 10^{-14} \mathrm{erg}$.

Thus we get the derived quantities:

$k_{B} T / 4 \pi \kappa=0.006$,

$\Sigma_{\text {crit }} \equiv \kappa / R_{0}{ }^{2}=1.2 \cdot 10^{-4} \mathrm{erg} \mathrm{cm}^{-2}$,

$\epsilon \equiv R_{0} \eta / b d^{2} \sim 0.5$

$\beta \equiv K_{0} d^{2} / \kappa \sim 3.5$.

Also, we estimated the induced tension $\Sigma \sim 2 \cdot 10^{-3} \mathrm{erg} \mathrm{cm}^{-2}$ in Sect. 3.2, so

$\Sigma / \eta \sim 2 \cdot 10^{3} \mu \mathrm{m} / \mathrm{sec}$ and the dimensionless tension

$\sigma \equiv \Sigma R_{0}^{2} / \kappa \sim 20$.

The values of $\Sigma, b, d$ are not known very accurately, but fortunately our formula for $k_{0}$ is not very sensitive to them.

\section{Appendix B. The Delaunay Surfaces}

We argued in the text against the introduction of a spontaneous curvature term in the elastic free energy. In the presence of such a term we may add and subtract a constant to recast the energy in the form

$$
F[\text { shape }]=2 \kappa \int\left[\left(H-H_{0}\right)^{2}+\sigma^{\prime}\right] .
$$

Large thermal fluctuations then imply that initially $\sigma^{\prime}=0$, and the free energy then simply prefers conformations with constant mean curvature everywhere equal to $H_{0}$.

Remarkably there is a one-parameter family of such shapes with axial symmetry. These are the "unduloid" or "Delaunay" surfaces. They obey a nonlinear equation expressing $H=H_{0}$. Deuling and Helfrich used this equation in ref. [32]. There is however a very simple geometrical characterization of these surfaces 46]: 
Draw a line $\ell$ in the plane. Construct any ellipse, tangent at one point to $\ell$. Let the ellipse roll without slipping along $\ell$, and follow the path of one focus to get a curve $C$. The figure of revolution obtained by rotating $C$ about the axis $\ell$ is a surface of constant mean curvature $1 / 2 r_{+}$, where $r_{+}$is the larger semimajor axis of the ellipse.

Let us see what this says for nearly-cylindrical shapes. Such shapes are generated from nearly-circular ellipses, with foci near the center. The radius $R_{0}$ of the near-cylinder is thus equal to that of the near-circle. Rolling the near-circle along the axis of course gives a periodic shape with period $\lambda=2 \pi R_{0}$. In our language, this is a small perturbation with wavenumber $k=1$, larger than the initial wavenumber seen experimentally.

\section{Appendix C. Thermal Fluctuations}

In subsection 3.3 we quoted an estimate for the effective stretching modulus of the bilayer.

Generally the effects of thermal fluctuations on the conformations of stiff membranes are quite small unless one carefully adjusts to the threshold of a shape transformation. One simply replaces the elastic constants by effective values corrected by terms of relative order $k_{B} T / 4 \pi \kappa$, about half a percent for DMPC. 15 We will neglect such effects. One notable exception, however, is the susceptibility $K^{-1}$, whose "bare" value is so small that thermal corrections can be significant. At modest values of tension, the membrane effectively becomes elastic, because significant area can hide in invisible short-scale wrinkles excited by thermal agitation [48] [7]. (The tiny value reported for the bare $K_{0}^{-1}$ in [7] is attained only at extremely large values of applied tension, where these wrinkles have been extinguished.)

Following Helfrich and Servuss, let us estimate the magnitude of this effect in our case. We consider a membrane thermally fluctuating about a cylindrical shape, and evaluate the average true area $A_{0} \equiv\left\langle\int \mathrm{d} S\right\rangle$ in terms of the "projected" or apparent area $2 \pi R_{0} L$. We would like to know how $A_{0}$ changes as we adjust the tension, at fixed projected area; this tells us how much area we can pull out of our membrane at fixed apparent shape, and hence the effective modulus. Following 44, the relative area extension as we change the dimensionless tension $\sigma$ is

$$
\delta A / A \sim \frac{k_{B} T}{8 \pi^{2}} \int_{\sqrt{\sigma_{0}}}^{\sqrt{\sigma}} \frac{\mathrm{d} k k^{2}}{\kappa k^{4}}
$$

15 Something similar happens with dynamic couplings 47 . 
In our case $\sigma \sim 20$ (Appendix A.). The lower bound of the integral should be either the initial tension or unity, whichever is larger, to account for the cylindrical geometry; since we take the initial tension to vanish and $\sigma \sim 20$ we get $\delta A / A \sim\left(k_{B} T / 8 \pi \kappa\right) \log 20 \sim 1 \%$. This estimate is not very sensitive to the actual value of $\sigma$. Then the effective modulus is given by the constitutive relation $\Sigma=K_{\text {eff }}(\delta A / A)$, or $K_{\text {eff }} \sim 100 \cdot \Sigma \sim 2 \cdot 10^{-1} \mathrm{erg} \mathrm{cm}^{-2}$, so that the hierarchy $(3.5)$ we assumed earlier is still preserved.

\section{Appendix D. Computation of the Dynamical Factor $\Lambda(k)$}

Eq. (6.3) is solved by separating variables as $\psi=\Psi(\rho) \exp \left(\omega t+i k z / R_{0}\right)$. $\Psi$ satisfies $\left(\partial_{\rho}^{2}-\rho^{-1} \partial_{\rho}-\left(k / R_{0}\right)^{2}\right)^{2} \Psi=0$, which has solutions of the form

$$
\Psi(\rho)=A_{1} \rho \mathrm{I}_{1}\left(k \rho / R_{0}\right)+B_{1} \rho \mathrm{K}_{1}\left(k \rho / R_{0}\right)+A_{2} \rho^{2} \mathrm{I}_{0}\left(k \rho / R_{0}\right)+B_{2} \rho^{2} \mathrm{~K}_{0}\left(k \rho / R_{0}\right)
$$

where $\mathrm{I}_{\nu}(x), \mathrm{K}_{\nu}(x)$ are modified Bessel functions [49]. Demanding that $\Psi(\rho)$ be wellbehaved at $\rho=0$ and $\rho=\infty$ forces $B_{1}=B_{2}=0$ in the interior fluid and $A_{1}=A_{2}=0$ in the exterior fluid. The no-slip boundary conditions, together with the lipid conservation law (4.2), give us four equations for the four unknowns $A_{1}, A_{2}, B_{1}, B_{2}$ :

$$
\begin{gathered}
v_{\rho}^{+}\left(\rho=R_{0}\right)=R_{0} \dot{u}, \quad v_{\rho}^{-}\left(\rho=R_{0}\right)=R_{0} \dot{u}, \\
v_{z}^{+}\left(\rho=R_{0}\right)=\tilde{v}=\frac{R_{0}}{i k} \dot{u}, \quad v_{z}^{-}\left(\rho=R_{0}\right)=\tilde{v}=\frac{R_{0}}{i k} \dot{u},
\end{gathered}
$$

where $+(-)$ denotes the exterior (interior) fluid. Note that in (D.3) we have assumed that the lipid velocity $\tilde{v}$ has no constant part, in accord with the arguments of Sect. 3 that after the tension has spread the $2 \mathrm{~d}$ pressure (tension) is practically uniform and we can take any net flow of lipid to be small. Also, we have taken the lipid to be incompressible since the applied tension is much smaller than the membrane compressibility (eqn. (3.5)). Plugging (D.1) into (D.2)-(D.3), and using the relations

$$
\begin{gathered}
\mathrm{I}_{0}^{\prime}(x)=\mathrm{I}_{1}(x), \quad \mathrm{I}_{1}^{\prime}(x)=\mathrm{I}_{0}(x)-\frac{1}{x} \mathrm{I}_{1}(x), \\
\mathrm{K}_{0}^{\prime}(x)=-\mathrm{K}_{1}(x), \quad \mathrm{K}_{1}^{\prime}(x)=-\mathrm{K}_{0}(x)-\frac{1}{x} \mathrm{~K}_{1}(x),
\end{gathered}
$$


we find

$$
\begin{aligned}
i \frac{k}{R_{0}}\left(A_{1} \mathrm{I}_{1}(k)+A_{2} R_{0} \mathrm{I}_{0}(k)\right) & =R_{0} \dot{u} \\
i \frac{k}{R_{0}}\left(B_{1} \mathrm{~K}_{1}(k)+B_{2} R_{0} \mathrm{~K}_{0}(k)\right) & =R_{0} \dot{u} \\
A_{1} \frac{k}{R_{0}} \mathrm{I}_{0}(k)+A_{2}\left(2 \mathrm{I}_{0}(k)+k \mathrm{I}_{1}(k)\right) & =\frac{R_{0}}{i k} \dot{u} \\
B_{1} \frac{k}{R_{0}} \mathrm{~K}_{0}(k)+B_{2}\left(k \mathrm{~K}_{1}(k)-2 \mathrm{~K}_{0}(k)\right) & =\frac{R_{0}}{i k} \dot{u},
\end{aligned}
$$

i.e. the $A$ 's and the $B$ 's are proportional to $\dot{u}$, with proportionality constants which are rational functions of the modified Bessel functions.

To relate $\dot{u}$ to $u$ and thus determine the growth rate $\dot{u} / u$, we examine the normal force balance equation at the membrane:

$$
T_{\rho \rho}^{+}-T_{\rho \rho}^{-}-\Delta p_{\text {Laplace }}=0
$$

where $T_{i j}$ is the $3 \mathrm{~d}$ stress tensor of water and $-\Delta p_{\text {Laplace }}$ is the pressure jump due to the surface tension, given by (4.1). The $z$ component of the Navier-Stokes equation relates the pressure to the velocity, $p^{ \pm}=\frac{\eta R_{0}}{i k} \nabla^{2} v_{z}^{ \pm}+p_{0}^{ \pm}$. The NS equation does not fix the constants $p_{0}^{ \pm} ;$we must choose $p_{0}^{+}=0$ and $p_{0}^{-}=\Sigma / R_{0}$. Using

$$
\left(\rho^{-1} \partial_{\rho} \rho \partial_{\rho}+\left(\frac{k}{R_{0}}\right)^{2}\right) f=0
$$

for $f(\rho)=\mathrm{I}_{0}\left(\frac{k \rho}{R_{0}}\right), \mathrm{K}_{0}\left(\frac{k \rho}{R_{0}}\right)$ [49, we find at the membrane 16

$$
\begin{aligned}
T_{\rho \rho}^{+}-T_{\rho \rho}^{-}= & -2 i \eta \frac{k}{R_{0}}\left(B_{1} \frac{k}{R_{0}}\left(\mathrm{~K}_{0}(k)+\frac{1}{k} \mathrm{~K}_{1}(k)\right)+B_{2} k \mathrm{~K}_{1}(k)\right) \\
& -2 i \eta \frac{k}{R_{0}}\left(A_{1} \frac{k}{R_{0}}\left(\mathrm{I}_{0}(k)-\frac{1}{k} \mathrm{I}_{1}(k)\right)+A_{2} k \mathrm{I}_{1}(k)\right)+\Sigma / R_{0} \\
\equiv & -\eta \Lambda(k)^{-1} \dot{u}+\Sigma / R_{0} .
\end{aligned}
$$

Eqn. (D.8b) defines what we call the "dynamical factor" $\Lambda(k)$ in Eq. (6.4). Taking the values for the $A$ 's and $B$ 's determined by (D.6), we find

$$
\Lambda(k)=-\frac{1}{2 k} \frac{\left[k\left(K_{0}^{2}-K_{1}^{2}\right)+2 K_{0} K_{1}\right]\left[k\left(I_{0}^{2}-I_{1}^{2}\right)-2 I_{0} I_{1}\right]}{3 I_{0} K_{0} / k-K_{1} I_{1} / k+k\left(I_{1}^{2} K_{0}^{2}-I_{0}^{2} K_{1}^{2}\right)} .
$$

In eqn. (D.9) all Bessel functions are to be evaluated at $k$.

16 We have used $T_{i j}=-p \delta_{i j}+\eta\left(\partial_{i} v_{j}+\partial_{j} v_{i}\right)$ for incompressible fluids [50]. 


\section{Appendix E. Membrane Elasticity}

Here we briefly derive eqn. (6.5). Following previous authors (e.g. [5] [6]) we visualize each monolayer as a fluid of compressible cylinders, each of length $D$. One end (the "chain") touches the bilayer midplane and resists compression and extension with a modulus $K_{c}$ and preferred area/molecule at the end of $a_{c 0}$. The other end (the "head") is a normal distance $\pm D$ from the midplane with modulus $K_{h}$ and preferred area $a_{h 0}$. All of the parameters introduced are the same for each monolayer, since the two layers and their respective solvents are identical. We thus consider a monolayer elastic energy per molecule of

$$
f^{ \pm}\left[\text {shape }, a_{h}^{ \pm}, a_{c}^{ \pm}\right]=\frac{1}{2} K_{h} a_{0 h}\left(\frac{a_{h}^{ \pm}}{a_{0 h}}-1\right)^{2}+\frac{1}{2} K_{c} a_{0 c}\left(\frac{a_{c}^{ \pm}}{a_{0 c}}-1\right)^{2}-\text { const. } .
$$

Here the constant includes the chemical potential for extracting additional molecules from any reservoir; it will of course turn out to be related to the physical tension.

It's convenient to introduce a pair of imaginary "neutral" surfaces at normal distance $\pm d$ from the midplane, where $d$ will be specified in a moment, and to measure the densities

$\phi^{ \pm}$of lipid molecules in the two monolayers relative to these surfaces. Thus the areas $a_{h, c}^{ \pm}$ above are

$$
\begin{aligned}
& a_{h}^{ \pm}=\left(\phi^{ \pm}\right)^{-1}\left[1 \mp 2 H^{ \pm}(D-d)\right] \\
& a_{c}^{ \pm}=\left(\phi^{ \pm}\right)^{-1}\left[1 \pm 2 H^{ \pm} d\right] .
\end{aligned}
$$

Here $H^{ \pm}$are the mean curvatures of the two neutral surfaces. We dropped terms involving Gauss curvature, as well as those of order $(H D)^{2}$. Substituting in (E.1) we get

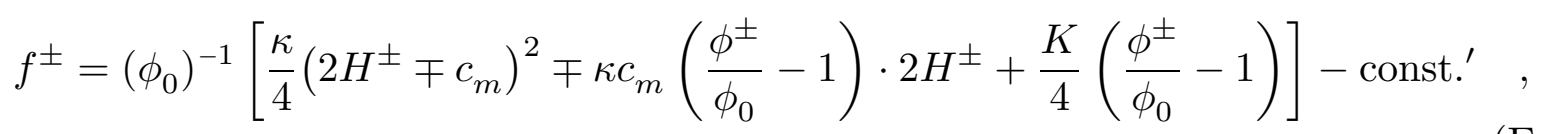

where $K, \phi_{0}, \kappa, c_{m}$, the new constant, and the neutral surface location $d$ are various combinations of the original parameters chosen to put (E.1) into the form (E.2). In particular $d$ is chosen to set the coefficient of the cross-term in the convenient form shown. Since the original parameters weren't directly observable anyway, we abandon them now in favor of the new ones. This is actually progress. Note that again the new parameters are the same for each monolayer. 
The monolayer free energy is now $\int \mathrm{d} S_{ \pm} \phi^{ \pm} f^{ \pm}$, where $\mathrm{d} S_{ \pm}$are the areas of the two neutral surfaces. Rewriting in terms of the area element $\mathrm{d} S$ of the midplane we get

$$
\begin{aligned}
& \mathrm{d} S_{ \pm} \phi^{ \pm} f^{ \pm}=\mathrm{d} S(1 \mp 2 H d) \\
& \times\left[\frac{\kappa \phi^{ \pm}}{4 \phi_{0}}\left(2 H^{ \pm}\right)^{2} \mp \frac{1}{2} \kappa c_{m}\left(2 H^{ \pm}\right)+\frac{\phi^{ \pm}}{\phi_{0}} \frac{K}{4}\left(\frac{\phi^{ \pm}}{\phi_{0}}-1\right)^{2}-\text { const. }^{\prime \prime} \cdot \phi^{ \pm}\right]
\end{aligned}
$$

Here $H$ is the mean curvature of the midplane: $H^{ \pm}=H \pm 2 H^{2} d$ plus a Gauss-curvature term we drop.

We will be considering only values of the tension much smaller than $K$ (eqn. (3.5)), so we may use $\phi_{0}$ in place of $\phi^{ \pm}$in the constant term above. Writing the other $H^{ \pm}$in terms of $H$ and finally identifying the constant with the tension $\Sigma$ then yields eqn. (6.5).

Before switching on the laser the terminal blobs serve as reservoirs, and we argued in the text that then $\Sigma$ should be taken to be close to zero. In equilibrium the densities will then just take their preferred values, and we recover the Canham-Helfrich model, with $\kappa$ the bilayer stiffness. In particular, no spontaneous curvature appears, as argued in [21]. After switching on the laser the system is no longer in equilibrium, and we need to work out dynamically what happens.

We note in passing that in a closed system the constant term in (E.1) will not be equal for the two layers; rather than containing a chemical potential for a reservoir, these terms act as Lagrange multipliers enforcing the constraint of fixed molecule numbers $N^{ \pm}$in each layer. Letting $A_{0}^{ \pm} \equiv a_{0} N^{ \pm}$be the "preferred area" of each layer, we then recover the area-difference elasticity model (ref. [6]). In particular the parameter $\alpha_{A D E}$ is essentially the same as our parameter $\beta$. 


\section{References}

[1] R. Lipowsky, "The conformation of membranes," Nature 349 (1991) 475.

[2] U. Seifert and R. Lipowsky, "Morphology of vesicles", in Structure and Dynamics of Membranes, ed. R. Lipowsky and E. Sackmann (Elsevier, 1995).

[3] J. Rothman, "Mechanisms of intracellular protein transport," Nature 372 (1994) 55.

[4] E. Gallez and W. Coakley, "Interfacial instability at cell membranes," Prog. Biophys. Molec Biol. 48 (1986) 155.

[5] U. Seifert, K. Berndl, and R. Lipowsky, "Shape transformation of vesicles," Phys. Rev. A44 (1991) 1182; L. Miao, B. Fourcade, M. Rao, M. Wortis, and R.K.P. Zia, "Equilibrium budding and vesiculation in the curvature model of fluid membranes," Phys. Rev. A43 (1991) 6843.

[6] L. Miao, U. Seifert, M. Wortis, and H.-G. Döbereiner, "Budding transitions of fluidbilayer vesicles," Phys. Rev. E49 (1994) 5389.

[7] E. Evans and W. Rawicz, "Entropy-driven tension and bending elasticity in condensedfluid membranes," Phys. Rev. Lett. 64 (1990) 2094.

[8] D. Needham, private communication.

[9] B. Pouligny, private communication; see also M. Angelova, B. Pouligny, G. MartinotLagarde, G. Grehan, and G. Gouesbet, "Stressing phospholipid membranes using mechanical effects of light," Prog. Colloid Polym. Sci. 97 (1994) 293, and in press.

[10] H. Miyata and H. Hotani, "Morphological changes in liposomes caused by polymerization of encapsulated actin and spontaneous formation of actin bundles," Proc. Nat. Acad. Sci. USA 89 (1992) 11547.

[11] D. Fygenson, private communication.

[12] R. Bar-Ziv and E. Moses, "Instability and 'pearling' states produced in tubular membranes by competition of curvature and tension," Phys. Rev. Lett., 73 (1994) 1392; R. Bar-Ziv and E. Moses, to appear.

[13] P. Nelson, T. Powers, and U. Seifert, "Dynamic theory of pearling instability in cylindrical vesicles," Phys. Rev. Lett. 74 (1995) 3384.

[14] J. Plateau, Statique experimentale et theorique des liquides soumis aux seules forces moleculaires (Gautier-Villars, 1873).

[15] Lord Rayleigh, "On the instability of jets," Proc. Lond. Math. Soc. 10 (1879) 4.

[16] Lord Rayleigh, "On the instability of a cylinder of viscous liquid under capillary force," Phil. Mag. 34 (1892) 145.

[17] S. Tomotika, "On the instability of a cylindrical thread of a viscous liquid surrounded by another viscous fluid," Proc. Roy. Soc. Lon. A150 (1932) 322.

[18] F. Brochard and J. Lennon, "Frequency spectrum in the flicker phenomenon in erythrocytes," J. Phys. France 36 (1975) 1035; M. Schneider, J. Jenkins, and W. Webb, "Thermal fluctuations of large cylindrical phospholipid bilayers," Biophys. J. 
45 (1984) 891; "Thermal fluctuations of large quasi-spherical bimolecular phospholipid vesicles," J. Phys. 45 (1984) 1457; S. Milner and S. Safran, "Dynamical fluctuations of droplet microemulsions and vesicles," Phys. Rev. A36 (1987) 4371.

[19] G. Dee and J. Langer, "Propagating pattern selection," Phys. Rev. Lett. 50 (1983) 383; E. Ben-Jacob, H. Brand, G. Dee, L. Kramer, and J. Langer, "Pattern propagation in nonlinear dissipative systems," Physica 14D (1985) 348; J. Langer, in Chance and matter, ed. J. Soulettie, J. Vannimenus, and R. Stora (North-Holland, 1987).

[20] W. van Sarloos, "Front propagation into unstable states," Phys. Rev. A37 (1988) 211.

[21] E. Evans, A, Yeung, R. Waugh, and J. Song, "Dynamic coupling and nonlocal curvature elasticity in bilayer membranes," in Structure and conformation of amphiphilic membranes, ed. R. Lipowsky et al (Springer, 1992).

[22] U. Seifert and S. Langer, "Viscous modes of bilayer membranes," Europh. Lett. 23 (1993) 71; "Hydrodynamics of membranes: the bilayer aspect and adhesion," Biophys. Chem. 49 (1994) 13.

[23] E. Evans and A. Yeung, "Hidden dynamics in rapid changes of bilayer shape," Chem. Phys. Lipids, 73 (1994) 39.

[24] R. Granek and Z. Olami, "Dynamics of Rayleigh-like instability induced by laser tweezers in tubular vesicles of self-assembled membranes," J. Phys. II France 5 (1995) 1348.

[25] S. Svetina, private communication.

[26] R. Bar-Ziv and E. Moses, to appear.

[27] E. Evans, Biophys. J., "Bending resistance and chemically induced moments in membrane bilayers," 14 (1974) 923; W. Helfrich, "Blocked lipid exchange in bilayers and its possible influence on the shape of vesicles," Z. Naturforsch. 29C (1974) 510.

[28] B. Thomas, private communication.

[29] S. Chiruvolu, H. Warriner, E. Naranjo, S. Idziak; J. Radler, R. Plano, J. Zasadzinski, and C. Safinya, "A phase of liposomes with entangled tubular vesicles," Science 266 (1994) 1222.

[30] S. Leibler, "Curvature instability in membranes," J. Phys. France 47 (1986) 507-516.

[31] U. Seifert, "Curvature-induced lateral phase separation in two-component vesicles," Phys. Rev. Lett. 70 (1993) 1335.

[32] H. Deuling and W. Helfrich, "A theoretical explanation for the myelin shapes of red blood cells," Blood Cells 3 (1977) 713; "The curvature elasticity of fluid membranes: a catalog of vesicle shapes," J. Phys. (Paris) 37 (1976) 1335.

[33] Ou-Yang Zhong-can and W. Helfrich, "Bending energy of vesicle membranes," Phys. Rev. A39 (1989) 5280.

[34] J. Israelachvili, Intermolecular and surface forces (Academic Press, 1992).

[35] J. D. Jackson, Classical electrodynamics (Wiley, 1975) pp. 107, 160. 
[36] R. Bar-Ziv, T. Frisch, and E. Moses, "Entropic expulsion in vesicles", preprint 1995 to appear in Phys. Rev. Lett.

[37] W. Wimley and T. Thompson, "Transbilayer and interbilayer phospholipid exchange in dimyristoylphosphatidylcholine/dimyristoylphosphatidylethanolamine large unilamellar vesicles," Biochemistry 30 (1991) 1702.

[38] R. Bar-Ziv, R. Menes, E. Moses, and S. Safran, "Local unbinding of pinched membranes," preprint 1995 to appear in Phys. Rev. Lett.

[39] G. Ahlers and D.S. Cannell, "Vortex-front propagation in rotating Couette-Taylor flow,"Phys. Rev. Lett. 50 (1983)1583; J. Fineberg and V. Steinberg, "Vortex-front propagation in Rayleigh-Bénard flow," Phys. Rev. Lett. 58 (1987) 1332.

[40] R. Waugh, J. Song, S. Svetina, and B. Zeks, "Local and nonlocal curvature elasticity in bilayer membranes by tether formation," Biophys. J. 61 (1992) 974.

[41] R.E. Goldstein, A.I. Pesci, and M.J. Shelley, "Topology Transitions and Singularities in Viscous Flow," Phys. Rev. Lett. 70, 3043 (1993); J. Eggers, "Universal pinching of 3D axisymmetric free-surface flow," Phys. Rev. Lett. 71, 3458 (1993); A.L. Bertozzi, M.P. Brenner, T.F. Dupont, and L.P. Kadanoff, "Singularities and similarities in interface flows," in Centennial Edition, Applied Mathematics Series, L. Sirovich, Ed. (Springer-Verlag Applied Mathematics Series, New York, 1993).

[42] X.D. Shi, M.P. Brenner, and S.R. Nagel, "A cascade of structure in a drop falling from a faucet," Science 265, 219 (1994).

[43] E. Evans and D. Needham, "Physical properties of surfactant bilayer membranes: thermal transitions, elasticity, rigidity, cohesion, and colloidal interactions," J. Phys. Chem. 91 (1987) 4219.

[44] D. Marsh, CRC handbook of lipid bilayers, (CRC, 1990) p. 166.

[45] R. Merkel, E. Sackmann, and E. Evans, "Molecular friction and epitactic coupling between monolayers in supported bilayers," J. Phys (Paris) 50 (1989) 1535.

[46] H. Bouasse, Cours de mathématiques générales (Paris, Delagrave, 1911).

[47] W. Cai and T.C. Lubensky, "Covariant hydrodynamics of fluid membranes," Phys. Rev. Lett. 73 (1994) 1186.

[48] W. Helfrich and R. Servuss, "Undulations, steric interaction and cohesion of fluid membranes," Nuovo Cimento 3D (1984) 137.

[49] M. Abramowitz and I. Stegun, eds., Handbook of mathematical functions (Dover, 1965).

[50] L. Landau and E. Lifsitz, Fluid mechanics (Pergamon, 1959). 